\title{
A NOTION OF RANK FOR UNITARY REPRESENTATIONS OF GENERAL LINEAR GROUPS
}

\author{
ROBERTO SCARAMUZZI
}

\begin{abstract}
A notion of rank for unitary representations of general linear groups over a locally compact, nondiscrete field is defined. Rank measures how singular a representation is, when restricted to the unipotent radical of a maximal parabolic subgroup. Irreducible representations of small rank are classified. It is shown how rank determines to a large extent the asymptotic behavior of matrix coefficients of the representations.
\end{abstract}

\section{DEFINITION AND BASIC PROPERTIES OF RANK}

\section{INTRODUCTION}

The unitary dual of $\mathrm{GL}_{n}(F), F$ a locally compact nondiscrete field, has been classified by D. Vogan [V] in the Archimedean case, and, up to the construction of all cuspidal representations, by M. Tadic in the non-Archimedean case.

Much remains to be done, however, in terms of the study of specific properties of the unitary representations involved. The inspiration for this paper comes form the following well-known theorem of Howe and Moore.

Theorem 0.1 [HM]. Let $G$ be a Zariski connected reductive algebraic group over a nondiscrete locally compact field $F$, and let $\rho$ be a unitary representation of dimension greater than 1 such that the center $Z$ of $G$ acts by a character. Then the absolute values of the matrix coefficients of $\rho$ approach zero at infinity on $G / Z$.

An easy consequence of this is

Corollary 0.2. In the hypotheses for Theorem 0.1 , if $N$ is a subgroup of $G$ which is not compact modulo $Z$, any vector that is invariant under $N$ transforms according to a character of $G$.

In particular if $\rho$ is irreducible and has a nontrivial $N$-invariant vector, $\rho$ must be a character.

Received by the editors August 15, 1988.

1980 Mathematics Subject Classification (1985 Revision). Primary 22E46, 22E50.

This paper was submitted, in a different form, in partial fulfillment of the requirements for Doctor of Philosophy in the Department of Mathematics of Yale University.

The author acknowledges support from the Consiglio Nazionale delle Ricerche, Italy. 
Hence it is possible to deduce a fact about a unitary representation of $G$ (whether it is one-dimensional or infinite-dimensional) just by looking at its $N$-spectrum.

The notion of rank of a unitary representation, first introduced by $\mathrm{R}$. Howe [H1] for symplectic groups, attempts to refine this idea.

Roughly said, the rank of a unitary representation of $\mathrm{GL}_{n}$ measures how singular a representation is, when restricted to the unipotent radical of a maximal parabolic subgroup.

In Chapter I of this paper, we show that rank is well defined for irreducible unitary representations of $\mathrm{GL}_{n}$.

We then proceed in Chapter II to classify unitary representations of small rank. If $\operatorname{rank} \rho=k<[n / 2]$, then $\rho$ is parabolically induced from the tensor product of an irreducible representation of $\mathrm{GL}_{n-k}$ with a character of $\mathrm{GL}_{k}$. It is worth noting that such a result is false for symplectic groups [H3].

The material in Chapter II is related, in a not readily apparent way, to the theory of reductive dual pairs [H2].

It turns out that the correspondence $\theta$ established in $\S I I .3$ is the same as the oscillator correspondence (between $\mathrm{GL}_{n}$ and $\mathrm{GL}_{k}$ ) when the character $\chi$ is fixed to be the trivial character.

The most important results are contained in Chapter III. We give estimates on the asymptotic decay of matrix coefficients of representations, based on their rank. These estimates refine and quantify Theorem 0.1 .

Theorem III.3.5 is a generalization of the result of D. A. Kazhdan [K] that the connected component of the trivial representation of $\mathrm{GL}_{n}, n \geq 3$, consists of characters. It states that for $k$ small enough, the set of representations of rank $k$ is both open and closed in $\mathrm{GL}_{n}$. We obtain as a byproduct a new and quantitative proof of Kazhdan's result.

The estimates we give can be usefully compared with some results of Langlands and Zuckerman [Z] on the asymptotics of matrix coefficients of admissible representations to obtain the nonunitarizability of certain induced $(\mathfrak{g}, K)$ modules [Sa1].

It is possible to compute the rank of a unitary representation in terms of the parameters appearing in the Tadić-Vogan classification, and thus obtain information about specific representations that are given in terms of these parameters.

This fits in better with the methods of a subsequent paper and we shall do it there.

\section{Notation}

The category of continuous representations of a locally compact group $G$, with intertwining operators as morphisms, will be denoted by $\operatorname{Rep} G$. The set of equivalence classes of irreducible unitary representations of $G$ will be denoted by $\widehat{G}$. We endow $\widehat{G}$ with the Fell topology [F1].

We shall often omit the adjective "unitary" in the sequel. 
We fix a locally compact nondiscrete field $F$ throughout this paper. Let $\mu$ be Haar measure on $F$. We shall denote by || an absolute value on $F$, normalized in such a way that if $E$ is a measurable subset of $F$ and $x \in F$,

$$
\mu(x E)=|x| \mu(E) .
$$

The group $\mathrm{GL}_{n}(F)$ will be denoted by $G_{n}$.

Let $k$ be an integer satisfying $0<k<n$. Then $P_{k}^{(n)}$ will denote the maximal parabolic subgroup of $G_{n}$ defined by

$$
P_{k}^{(n)}=\left\{g \in G_{n}, g_{i j}=0 \text { if } i>k \text { and } j \leq k\right\} .
$$

The group $P_{k}^{(n)}$ is the stabilizer of the span of the first $k$ basis vectors in the standard left action of $G_{n}$ on $F^{n}$.

The unipotent radical of $P_{k}^{(n)}$ will be denoted by $N_{k}^{(n)}$. Namely

$$
N_{k}^{(n)}=\left\{g \in G_{n}, g_{i j}=\delta_{i j} \text { if } i>k \text { or } j \leq k\right\} .
$$

Note that $N_{k}^{(n)}$ is abelian and naturally isomorphic to $\operatorname{Hom}_{F}\left(F^{n-k}, F^{k}\right)$.

The Levi component of $P_{k}^{(n)}$ will be denoted by $L_{k}^{(n)}$ :

$$
L_{k}^{(n)}=P_{k}^{(n)} \cap\left(P_{k}^{(n)}\right)^{t} \text {, }
$$

where $t$ denotes the transpose operation on matrices. Note that $L_{k}^{(n)} \simeq G_{k} \times$ $G_{n-k}$. Also $P_{k}^{(n)}$ is a semidirect product

$$
P_{k}^{(n)}=L_{k}^{(n)} \ltimes N_{k}^{(n)}
$$

and the action by conjugation of $L_{k}^{(n)}$ on $N_{k}^{(n)}$ becomes the standard action of $G_{k} \times G_{n-k}$ on $\operatorname{Hom}_{F}\left(F^{n-k}, F^{k}\right)$ by pre- and post-multiplication:

$$
T \cdot(A, B)=A^{-1} T B \text {. }
$$

We shall also denote the parabolic subgroup $P_{k}^{(n)} \cap P_{n-k}^{(n)}$ by $Q_{k}^{(n)}$ and its unipotent radical by $H_{k}^{(n)}$.

We shall omit, for all the groups just defined, the superscript ${ }^{(n)}$ when no confusion can arise.

\section{RANK FOR REPRESENTATIONS OF $P_{k}^{(n)}$}

We fix, for the rest of this paper, a nontrivial character $\chi_{1}$ of $F$. We can now identify $\left(N_{k}^{(n)}\right)^{\wedge}$ with $\operatorname{Hom}_{F}\left(F^{k}, F^{n-k}\right)=\left(N_{k}^{(n)}\right)^{*}$ as follows: if $n^{*} \in$ $\operatorname{Hom}\left(F^{k}, F^{n-k}\right)$ and $n \in \operatorname{Hom}_{F}\left(F^{n-k}, F^{k}\right)$, define $\chi_{n^{*}} \in\left(N_{k}^{(n)}\right)^{\wedge}$ by

$$
\chi_{n^{*}}(n)=\chi_{1}\left(\operatorname{tr}\left(n^{*} \circ n\right)\right) \text {. }
$$

The adjoint action on $\left(N_{k}^{(n)}\right)^{\wedge}$ induced by the action by conjugation of $L_{k}^{(n)}$ on $N_{k}^{(n)}$ now corresponds to the standard action of $G_{k} \times G_{n-k}$ on $\operatorname{Hom}_{F}\left(F^{k}, F^{n-k}\right)$. 
In particular the $L_{k}^{(n)}$-orbits on $\left(N_{k}^{(n)}\right)^{\wedge}$ are

$$
\mathscr{O}_{l}=\left\{n^{*}, n^{*} \text { is a linear transformation of rank } l\right\}
$$

for integers $l$ satisfying $0<l \leq \min (k, n-k)$.

The groups $N_{k}^{(n)}$ and $P_{k}^{(n)}$ are well known to be of type I and it is easily seen that the $\mathscr{O}_{l}$ are locally closed subsets $\left(N_{k}^{(n)}\right)^{\wedge}$. Hence the extension $1 \rightarrow$ $N_{k}^{(n)} \rightarrow P_{k}^{(n)} \rightarrow L_{k}^{(n)} \rightarrow 1$ satisfies the hypotheses for Mackey theory [R].

Let $\rho \in \operatorname{Rep} N_{k}^{(n)}$. Write

$$
\rho \simeq \int_{\left(N_{k}^{(n)}\right)^{*}}^{\oplus} m_{n^{*}} \chi_{n^{*}} d \mu\left(n^{*}\right)
$$

for some multiplicity function $m$ and some Borel measure $\mu$ on $\left(N_{k}^{(n)}\right)^{*}$.

If $E \subseteq\left(N_{k}^{(n)}\right)^{*}$ is measurable, set $\mu_{i}(E)=\mu\left(E \cap \mathscr{O}_{i}\right)$. Then

$$
\mu=\mu_{0} \oplus \mu_{1} \oplus \cdots \oplus \mu_{\min (k, n-k)} .
$$

Also

$$
\rho=\rho_{0} \oplus \cdots \oplus \rho_{\min (k, n-k)},
$$

where

$$
\rho_{i}=\int_{\left(N_{k}^{(n)}\right)^{*}}^{\oplus} m_{n^{*}} \chi_{n^{*}} d \mu_{i}\left(n^{*}\right) .
$$

If $\rho$ is actually the restriction of a representation of $P_{k}^{(n)}$, it is clear that $\rho=$ $\rho_{0} \oplus \cdots \oplus \rho_{\min (k, n-k)}$ also as a representation of $P_{k}^{(n)}$.

Definition 2.1. A unitary representation $\rho$ of $P_{k}^{(n)}$ has pure rank $r$ if $\rho_{m}=0$ for all $m \neq r$.

In particular representations of $P_{k}^{(n)}$ have pure rank.

\section{THE RANK OF A REPRESENTATION OF $\mathrm{GL}_{n}$}

Definition 3.1. Let $\rho \in \operatorname{Rep} G_{n}$. We say $\rho$ has pure $N_{k}$-rank if $\left.\rho\right|_{P_{k}^{(n)}}$ has pure rank $r$.

Theorem 3.2. Let $\rho \in \operatorname{Rep} G_{n}$ and suppose $\rho$ has pure $N_{k}$-rank $r$ and pure $N_{l}$-rank $s$. Then

(1) If both $r$ and $s$ are $\leq \min (k, l, n-k, n-l)$, then $r=s$.

(2) If $r>\min (l, n-l)$, then $s=\min (l, n-l)$.

Corollary 3.3. Suppose $\rho \in \operatorname{Rep} G_{n}$ has pure $N_{k}$-rank for all $k$. Then

$$
N_{k}-\operatorname{rk}(\rho)=\min \left(k, n-k, N_{[n / 2]}-\operatorname{rk} \rho\right) .
$$

Hence the subgroup $N_{[n / 2]}$ detects "all rank there is". 
Proof of Theorem 3.2. Let $\phi: A \rightarrow B$ be a morphism of abelian groups. Then we have an adjoint map $\hat{\phi}: \operatorname{Rep} B \rightarrow \operatorname{Rep} A$ given by $\hat{\phi}(\pi)=\pi \circ \phi$. Note that $\hat{\phi}$ maps $\widehat{B}$ to $\widehat{A}$. If $\mu$ is a measure on $\widehat{B}, \hat{\phi}_{*}(\mu)$ is a measure on $\widehat{A}$. If $\pi \in \operatorname{Rep} B$ has spectral measure $\mu$, then $\hat{\phi}(\pi)$ has spectral measure $\hat{\phi}_{*}(\mu)$.

Let $Z=N_{k} \cap N_{l}$. Then $Z$ is a normal subgroup of $P_{k} \cap P_{l}$. It can be identified naturally with $\operatorname{Hom}_{F}\left(F^{\min (n-k, n-l)}, F^{\min (k, l)}\right)$. As in $\S 1, \widehat{Z}$ can be identified with $Z^{*}=\operatorname{Hom}_{F}\left(F^{\min (k, l)}, F^{\min (n-k, n-l)}\right)$.

The injections $i_{k}: Z \rightarrow N_{k}$ and $i_{l}: Z \rightarrow N_{l}$ induce projections $p_{k}: N_{k}^{*} \rightarrow Z^{*}$ and $p_{l}: N_{l}^{*} \rightarrow Z^{*}$. Under our identifications these correspond to the maps $\hat{i}_{k}: \widehat{N}_{k} \rightarrow \widehat{Z}$ and $\hat{i}_{l}: \widehat{N}_{l} \rightarrow \widehat{Z}$ defined above.

The $P_{k} \cap P_{l}$ orbits on $Z^{*}$ are $\mathscr{O}_{t}^{\prime}$ for $0 \leq t \leq \min (k, l, n-k, n-l)$, where

$$
\mathscr{\sigma}_{t}^{\prime}=\left\{z^{*}, \text { the rank of the linear transformation } z^{*} \text { is } t\right\} .
$$

Note that there is only one quasi-invariant measure class on $\mathscr{O}_{r}^{(k)}$, the $P_{k}$ orbit in $N_{k}^{*}$ consisting of linear transformations of rank $k$, namely the class of Lebesgue measure.

Hence the spectral measures $\mu_{k}$ and $\mu_{l}$ of $\left.\rho\right|_{N_{k}}$ and $\left.\rho\right|_{N_{l}}$ are (equivalent to) respectively Lebesgue measure on $\mathscr{O}_{r}^{(k)}$ and $\mathscr{O}_{s}^{(l)}$.

Suppose $r \leq \min (k, l, n-k, n-l)$. If $n^{*} \in \mathscr{O}_{r}^{(k)}$, then certainly $\operatorname{rk}\left(p_{k} n^{*}\right) \leq$ $r$. Furthermore the condition for $\operatorname{rk}\left(p_{k} n^{*}\right)<r$ is that $n^{*}$ satisfy a set of nontrivial polynomial equations. Hence the set $\left\{n^{*} \in \mathscr{O}_{r}^{(k)}, \operatorname{rk}\left(p_{k} n^{*}\right)<r\right\}$ has Lebesgue measure zero in $\mathscr{O}_{r}^{(k)}$. Analogously, if $r>\min (k, l, n-k, n-l)$, almost all $p_{k} n^{*}$ lie in $\mathscr{O}_{\min (k, l, n-k, n-l)}^{\prime}$. We see that

$$
\left(p_{k}\right)_{*}\left(\mu_{k}\right)=\left\{\begin{array}{l}
\text { Lebesgue measure on } \mathscr{O}_{r}^{\prime} \text { if } r \leq \min (k, l, n-k, n-l), \\
\text { Lebesgue measure on } \mathscr{O}_{\min (k, l, n-k, n-l)}^{\prime} \text { otherwise }
\end{array}\right.
$$

and analogously for $\left(p_{l}\right)_{*}\left(\mu_{l}\right)$.

The spectral measure $\left.\rho\right|_{Z}$ must be (equivalent to) both $\left(p_{k}\right)_{*}\left(\mu_{k}\right)$ and $\left(p_{l}\right)_{*}\left(\mu_{l}\right)$. This clearly implies the theorem.

Definition 3.4. Suppose that $\rho \in \operatorname{Rep} G_{n}$ has pure $N_{k}$-rank for all $k$. Then the $N_{[n / 2]}$-rank of $\rho$ is called the $N$-rank of $\rho$ and denoted by rk $\rho$.

Lemma 3.5. Let $\rho \in \operatorname{Rep} G_{n}$. Then the following are equivalent.

(1) $\rho$ has pure $N_{k}$-rank zero for some $k$.

(2) $\rho$ has $N$-rank zero.

(3) $\rho$ is a direct integral of characters of $G_{n}$.

Proof. (3) implies (2) since all characters of $G_{n}$ have the form $\chi \circ$ det for some character $\chi$ of $F^{x}$, the group of nonzero elements of the base field, hence are trivial on $N_{[n / 2]}$. (2) implies (1) trivially. It follows easily from Corollary 0.2 that (1) implies (3). 
The preceding lemma is essentially a restatement of Theorem 0.1 . It can be seen as a particular case of the following theorem.

Theorem 3.6. Let $\rho \in \operatorname{Rep} G_{n}$ and let $\left.\rho\right|_{P_{l}}=\rho_{0} \oplus \cdots \oplus \rho_{\min (l, n-l)}$, be the decomposition of $\left.\rho\right|_{P_{l}}$ into subrepresentations of fixed $N_{l}$-rank. Let $\mathscr{H}_{k}$ be the representation space of $\rho_{k}$. Then for every $k, \mathscr{H}_{k}$ is $\rho\left(G_{n}\right)$-invariant. In particular if $\rho$ is irreducible, $\rho$ has pure $N_{k}$-rank. Thus we have the decomposition

$$
\widehat{G}_{n}=\bigcup_{0 \leq k \leq[n / 2]}\left(\widehat{G}_{n}\right)_{k},
$$

where $\left(\widehat{G}_{n}\right)_{k}$ is the set of irreducible representations of $G_{n}$ of $N$-rank $k$.

An essential part of the proof will be a knowledge of the representation theory of the Heisenberg group $H_{1}^{(n)}=N_{1}^{(n)} N_{n-1}^{(n)}$. Write $H_{1}^{(n)}=\{(x, y, z), x \in$ $\left.\left(F^{n-2}\right)^{*}, y \in F^{n-2}, z \in F\right\}$ corresponding to

$$
H_{1}^{(n)}=\left\{\left[\begin{array}{lll}
1 & x & z \\
0 & I & y \\
0 & 0 & 1
\end{array}\right]\right\} \text {. }
$$

The center of $H_{1}^{(n)}$ is $Z=\{(0,0, z)\}$. We have

Theorem 3.7 (Stone-Von Neumann [VN]). $\widehat{H}_{1}^{(n)}$ can be described as follows: If $\rho \in \widehat{H}_{1}^{(n)}, Z$ acts by a character $\chi$. Then

(1) If $\chi$ is the trivial character, $\rho$ is a character of $H_{1}^{(n)} / Z \simeq F^{2(n-2)}$.

(2) Let $\chi$ be a nontrivial character of $Z$. Then there is exactly one representation $\rho_{\chi}$ in $\widehat{H}_{1}^{(n)}$ with central character $\chi$. Let $X=\{(x, 0,0)\}$.

Then $\rho_{\chi}$ can be realized on $L^{2}(X)$ by the following formulae:

$$
\begin{aligned}
& \left(\rho_{\chi}(x, 0,0) f\right)\left(x^{\prime}\right)=f\left(x+x^{\prime}\right), \\
& \left(\rho_{\chi}(0, y, 0) f\right)\left(x^{\prime}\right)=\chi\left(x^{\prime} y\right) f\left(x^{\prime}\right), \\
& \left(\rho_{\chi}(0,0, z) f\right)\left(x^{\prime}\right)=\chi(z) f\left(x^{\prime}\right) .
\end{aligned}
$$

Proof of Theorem 3.6. We shall prove, by induction on $n$, that $\mathscr{H}_{k}$ is $\rho\left(G_{n}\right)$ invariant if $k<\min (l, n-l)$.

If $n=2$ or 3 the only possible case is $k=0$. The required result is Lemma 3.5.

Now let $n>3$. If $k=0$ we are again done by Lemma 3.5. If $l=1$ or $n-1, k=0$ is the only possible case, so we may assume $1<l<n-1$. In this case $P_{l}$ and $Q_{1}=P_{1} \cap P_{n-1}$ generate $G_{n}$, so that it is sufficient to prove that $\mathscr{H}_{k}$ is $\rho\left(Q_{1}\right)$-invariant. We have the Levi decomposition

$$
Q_{1}=M \ltimes H^{(n)},
$$

where $M \simeq F^{\times} \times G_{n-2} \times F^{\times}$. 
We examine the representation theory of $Q_{1}$. The action of $Q_{1}$ on $\hat{Z}$, the dual of the center $Z$ of $H_{1}^{(n)}$, has exactly two orbits: \{trivial character\} and $\hat{Z}$-\{trivial character $\}$. The action is the standard action of $G_{1} \times G_{1}$ on $\operatorname{Hom}_{F}(F, F)$. Fix a nontrivial character $\chi_{1}$ of $Z$. Let

$$
J=\left\{g \in Q_{1}, g_{11}=g_{n n}\right\} .
$$

Then $J=\operatorname{stab}_{Q_{1}}\left(\chi_{1}\right)$ and $[R]$ tells us that $\operatorname{Ind}_{J}^{Q_{1}}$ is an equivalence of categories between the category of representations of $Q_{1}$ with no $Z$-invariant vectors and the category $(\operatorname{Rep} J)_{1}$ of representations of $J$ which restrict to a multiple of $\chi_{1}$ on $Z$.

Theorem 3.7 tells us there is exactly one irreducible representation $\rho_{1}$ of $H_{1}^{(n)}$ which restricts to a multiple of $\chi_{1}$ on $Z$.

It is very easy to check that $\rho_{1}$ extends to the representation $\omega_{1}$ of $J$ defined as follows: Let $M_{1}=J \cap M$,

$$
M_{1}=\left\{\operatorname{diag}(\alpha, A, \alpha), \alpha \in F^{\times}, A \in G_{n-2}\right\} .
$$

Then

$$
J=M_{1} \ltimes H_{1}^{(n)} .
$$

Define

$$
\left(\omega_{1}(\alpha, A, \alpha) f\right)\left(x^{\prime}\right)=|\alpha|^{(2-n) / 2}|\operatorname{det} A|^{1 / 2} f\left(\alpha^{-1} x^{\prime} A\right)
$$

and

$$
\omega_{1} \mid H_{1}^{(n)}=\rho_{1} .
$$

Again $[\mathrm{R}]$ says that $\_\omega_{1}$ is an equivalence of categories between $\operatorname{Rep} M_{1}$ and $(\operatorname{Rep} J)_{1}$.

In conclusion, if $\rho \in \operatorname{Rep} G_{n}$ has no $Z$-invariant vectors, then $\left.\rho\right|_{Q} \simeq$ $\operatorname{Ind}_{J}^{Q} \tau \otimes \omega_{1}$ and the structure of $\left.\rho\right|_{Q}$ is the same as the structure of $\tau$. By induction, $\left.\tau\right|_{G_{n-2}}$ satisfies the theorem. It suffices to show that if $\left.\tau\right|_{G_{n-2}}$ has pure rank, then $\rho$ also has pure rank and $\operatorname{rk} \rho=\left.\operatorname{rk} \tau\right|_{G_{n-2}}+1$.

To this end we analyze the spectrum of $\left.\rho\right|_{N_{l}}$. The group $N_{l}$ is a normal subgroup of $P_{l} \cap Q_{1}$. The action by conjugation of $P_{l} \cap Q_{1}$ on $N_{l}$ induces a map $P_{l} \cap Q_{1} \rightarrow G_{l} \times G_{n-l}$. The image of $P_{l} \cap Q_{1}$ is $P_{1}^{(l)} \times P_{n-l-1}^{(n-l)}$. The group $P_{1}^{(l)}$ is the stabilizer in $G_{l}$ of $F e_{1}$ and $P_{n-l-1}^{(n-1)}$ is the stabilizer in $G_{n-l}$ of $V_{n-l-1}=\operatorname{span}\left\{e_{l+1} \cdots e_{n-1}\right\}$.

The $\left(G_{l} \times G_{n-l}\right)$-orbit $\mathscr{O}_{k} \subseteq N_{l}^{*}$ decomposes under the action of $P_{1}^{(l)} \times P_{n-l-1}^{(n-l)}$ into the following orbits:

$$
\begin{aligned}
& \mathscr{O}_{k,-, i}=\left\{n^{*}, \operatorname{rk} n^{*}=k, n^{*}\left(e_{1}\right)=0, \operatorname{dim}\left(\operatorname{Im} n^{*} \cap V_{n-l-2}\right)=i\right\}, \\
& \mathscr{O}_{k, *, i}=\left\{n^{*}, \operatorname{rk} n^{*}=k, n^{*}\left(e_{1}\right) \neq 0, n^{*}\left(e_{1}\right) \in V_{n-l-1},\right. \\
& \left.\quad \operatorname{dim}\left(\operatorname{Im} n^{*} \cap V_{n-l-1}\right)=i\right\}, \\
& \mathscr{O}_{k,+, i}=\left\{n^{*}, \operatorname{rk} n^{*}=k, n^{*}\left(e_{1}\right) \notin V_{n-l-1}, \operatorname{dim}\left(\operatorname{Im} n^{*} \cap V_{n-l-1}\right)=i\right\} .
\end{aligned}
$$


Write $\tau$ as a direct integral of irreducible representations of $M$. Almost all representations appearing in the direct integral decomposition of $\tau$ have pure rank equal to that of $\left.\tau\right|_{G_{n-2}}$ when restricted to $G_{n-2}$. The functor $\operatorname{Ind}_{J-}^{Q} \otimes \omega_{1}$ preserves direct integrals so we an assume $\tau$ to be irreducible.

In this case $\tau=\pi \otimes \chi$ where $\pi=\left.\tau\right|_{G_{n-2}}$ and $\chi \in\left(M_{1} / G_{n-2}\right)^{\wedge}=\widehat{F}^{\times}$. Let $\sigma=\operatorname{Ind}_{J}^{Q_{1}} \pi \otimes \chi \otimes \omega_{1}$.

Since we can choose representatives for $J \backslash Q_{1}$ which centralize $M_{1}$, the Mackey decomposition theorem [M2] shows

$$
\sigma=\pi \otimes \chi \otimes \operatorname{Ind}_{J}^{Q_{1}} \omega_{1} .
$$

The orbit $\mathscr{O}_{k,-, k}$ in $N_{l}^{*}$ consists of those characters of $N_{l}$ which factor to $N_{l} \cap G_{n-2}$. Hence the $N_{l}$-spectral measure of $\pi$ is concentrated on $\mathscr{O}_{\text {rk } \pi,-, \text { rk } \pi}$.

We next want to show that $\operatorname{Ind}_{J}^{Q_{1}} \omega_{1}$ has spectral measure concentrated on $\mathscr{O}_{1,+, 0}$. Indeed, first consider $\omega_{1}$. If $g \in N_{l}$, write $g$ as

$$
g=\left[\begin{array}{c|c|c}
I & x & z \\
\hline 0 & & \multicolumn{2}{|c}{I}
\end{array}\right] .
$$

If $x^{\prime} \in X$, write $x^{\prime}=\left(x_{1}, x_{2}\right)$ with $x_{1} \in\left(F^{l-1}\right)^{*}, x_{2} \in\left(F^{n-l-1}\right)^{*}$. Then, with obvious notation,

$$
\left(\omega_{1}(x, y, z, B) f\right)\left(x_{1}, x_{2}\right)=\chi_{1}(z) \chi_{1}\left(x_{2}, y\right) f\left(x, x_{1} B+x_{2}+x\right) .
$$

Let $U$ be the operator consisting of the Fourier transform with respect to $x_{2}$. Precisely, if $\xi$ denotes an $(n-l-1)$-column vector, then

$$
(U f)\left(x_{1}, \xi\right)=\int_{F^{n-l-1}} f\left(x_{1}, x_{2}\right) \chi_{1}\left(-x_{2} \xi\right) d x_{2} .
$$

Then it is easy to compute that

(28) $\left(U \omega_{1}(x, y, z, B) U^{-1} \phi\right)\left(x_{1}, \xi\right)=\chi_{1}(z) \chi_{1}\left(x_{1} y\right) \chi_{1}\left(x_{1} B \xi\right) \chi_{1}(x \xi) \phi\left(x_{1}, \xi\right)$.

We have just written $\omega_{1}$ as a direct integral

$$
\omega_{1} \simeq \int_{F^{1-1} \times F^{n-1-1}}^{\otimes} \chi_{x_{1}, \xi} d \mu\left(x_{1}, \xi\right),
$$

where

$$
\chi_{x_{1}, \xi}(x, y, z, B)=\chi_{1}(z) \chi_{1}\left(x_{1} y\right) \chi_{1}\left(x_{1} B \xi\right) \chi_{1}(x \xi) .
$$

Under our identifications $\chi_{x_{1}, \xi}$ corresponds to the matrix $\left[\begin{array}{cc}x_{1} & 1 \\ \xi x_{1} & \xi\end{array}\right]$ which lies in $\mathscr{O}_{1,+, 0}$.

We now turn to the effect of $\operatorname{Ind}_{J}^{Q_{1}}$ on the $N_{l}$-spectrum of a representation. Let $\nu \in \operatorname{Rep} J$ and let

$$
\left.\nu\right|_{N_{l}} \simeq \int_{N_{l}^{*}}^{\oplus} m_{n^{*}} \chi_{n^{*}} d \mu\left(n^{*}\right)
$$


We have

$$
J \backslash Q_{1} \simeq \frac{F^{\times} \times F^{\times}}{\operatorname{diag}} .
$$

If $(\alpha, \beta) \in F^{\times} \times F^{\times}$, set

$$
n^{*} \cdot(\alpha, \beta)=\left[\begin{array}{ll}
\alpha & 0 \\
0 & I
\end{array}\right]^{-1} n^{*}\left[\begin{array}{ll}
I & 0 \\
0 & \beta
\end{array}\right] \text {. }
$$

Let $\mu \cdot(\alpha, \beta)$ and $m_{n^{*}} \cdot(\alpha, \beta)$ be induced by this action. Let $d(\overline{\alpha, \beta})$ be Lebesgue measure on $\left(F^{\times} \times F^{\times}\right) /$diag. Then Mackey decomposition [M2] tells us that

$$
\left.\left(\operatorname{Ind}_{J}^{Q_{1}} \nu\right)\right|_{N_{l}}=\int_{\left(F^{\times} \times F^{\times}\right) / \text {diag }}^{\oplus} \int_{N_{l}^{*}}^{\oplus} m_{n^{*}} \cdot(\alpha, \beta) \chi_{n^{*}} d(\mu \cdot(\alpha, \beta))\left(n^{*}\right) d(\overline{\alpha, \beta}) .
$$

This discussion, applied to $\nu=\omega_{1}$, shows that the $N_{l}$-spectral measure of Ind $Q_{J} \omega_{1}$ is concentrated on $\mathscr{O}_{1,+, 0}$.

Finally we show that the spectral measure of $\sigma=\pi \otimes \chi \otimes \operatorname{Ind}_{J}^{Q} \omega$ is concentrated on $\mathscr{O}_{\mathrm{rk} \pi+1}$. Indeed $\mathscr{O}_{1,+, 0}+\mathscr{O}_{\mathrm{rk} \pi,-, \mathrm{rk} \pi}$ is Zariski open (hence of total measure) in $\mathscr{O}_{\mathrm{rk} \pi+1}$.

Let $\pi^{\prime}$ and $\pi^{\prime \prime}$ be two representations of $N_{l}$. Consider the (outer) tensor product $\pi^{\prime} \otimes \pi^{\prime \prime}$ of $\pi^{\prime}$ and $\pi^{\prime \prime}$. This is a representation of $N_{l} \times N_{l}$. Its spectrum is the Cartesian product of the spectra of $\pi^{\prime}$ and $\pi^{\prime \prime}$. Taking the inner tensor product amounts to taking the restriction to the diagonal of the outer tensor product. If diag: $N_{l} \rightarrow N_{l} \times N_{l}$, let diag*: $N_{l}^{*} \times N_{l}^{*} \rightarrow N_{l}^{*}$ be the adjoint map.

We have

$$
\operatorname{diag}^{*}\left(n_{1}^{*}, n_{2}^{*}\right)=n_{1}^{*}+n_{2}^{*}
$$

Hence

$$
\operatorname{diag}^{*}\left(\mathscr{O}_{1,+, 0} \times \mathscr{O}_{\mathrm{rk} \pi,-, \mathrm{rk} \pi}\right)=\mathscr{O}_{1,+, 0}+\mathscr{O}_{\mathrm{rk} \pi,-, \mathrm{rk} \pi} \cdot
$$

This concludes the proof of Theorem 3.6.

\section{CLASSIFICATION OF REPRESENTATIONS OF SMALL RANK}

\section{IRREDUCIBILITY PROPERTIES}

The main result of this section is

Theorem 1.1. Let $\rho, \rho^{\prime} \in\left(\widehat{G}_{n}\right)_{k}, k<[n / 2]$, and $l \geq k$. Then $\operatorname{Hom}_{G_{n}}\left(\rho, \rho^{\prime}\right)=$ $\operatorname{Hom}_{Q_{l}(n)}\left(\rho, \rho^{\prime}\right)$. In particular $\left.\rho\right|_{Q_{l}}$ is irreducible and if $\rho \simeq \rho^{\prime}$, then $\left.\rho\right|_{Q_{l}} \simeq$ $\left.\rho^{\prime}\right|_{Q_{l}}$, so that restriction to $Q_{l}$ defines an injection $\left(\widehat{G}_{n}\right)_{k} \rightarrow \widehat{Q}_{l}^{(n)}$.

Before passing to the proof, we wish to discuss a certain representation of $G_{2}$. We define $\omega_{1}^{(2)} \in \operatorname{Rep} G_{2}$ as follows: Its representation space is $L^{2}\left(F^{2}\right)$ and if $g \in G_{2}, f \in L^{2}\left(F^{2}\right)$ and $x \in F^{2}$, we define

$$
\left(\omega_{1}^{(2)}(g) f\right)(x)=|\operatorname{det} g|^{1 / 2} f(x g) \text {. }
$$


Lemma 1.2. (a) $\operatorname{Hom}_{G_{2}}\left(\omega_{1}^{(2)}, \omega_{1}^{(2)}\right)=\operatorname{Hom}_{Q_{1}^{(2)}}\left(\omega_{1}^{(2)}, \omega_{1}^{(2)}\right)$.

(b) Let $\lambda$ and $\lambda^{\prime}$ be characters of $G_{2}$. Then if $\lambda \neq \lambda^{\prime}$, the intersection of the spectra of $\lambda \otimes \omega_{1}^{(2)}$ and $\lambda^{\prime} \otimes \omega_{1}^{(2)}$ has zero measure in both spectra. In particular $\operatorname{Hom}_{G_{2}}\left(\lambda \otimes \omega_{1}^{(2)}, \lambda^{\prime} \otimes \omega_{1}^{(2)}\right)=\{0\}$.

Proof. For the purposes of this proof we abbreviate $G_{2}$ to $G, Q_{1}^{(2)}$ to $Q$, and $\omega_{1}^{(2)}$ to $\omega$. Let $N$ be the unipotent radical of $Q$ and $A$ its Levi component. Also, let $Z$ denote the center of $G$. If we apply the Mackey theory of representations of group extensions [R] to $Q=A \ltimes N$ we obtain that the irreducible representations of $Q$ are:

(a) characters of $A$;

(b) $\operatorname{Ind}_{Z N}^{Q} \chi \otimes \chi_{1}, \chi \in \hat{Z}, \chi_{1}$ a fixed character of $N$.

In particular, if $\rho \in \operatorname{Rep} Q$ and $\left.\rho\right|_{N}$ is equivalent to the regular representation of $N$, then $\rho$ is irreducible.

Also note that

$$
\left.\left(\operatorname{Ind}_{Z N}^{Q} \chi \otimes \chi_{1}\right)\right|_{Z}=\infty \chi .
$$

As a measure space, $F^{2} \simeq F^{2}-\{0\}$. $G$ acts transitively on $F^{2}-\{0\}$. The stabilizer of $(0,1)$ is

$$
s=\left\{\left[\begin{array}{cc}
x & y \\
0 & 1
\end{array}\right], x \in F^{\times}, y \in F\right\} .
$$

Hence, as homogeneous spaces, $F^{2}-\{0\} \simeq S \backslash G$ and $\omega$ can be viewed as the regular representation of $G$ on $L^{2}(S \backslash G)$. We have

$$
\omega=\operatorname{Ind}_{S}^{G} 1_{S},
$$

where $1_{S}$ denotes the trivial representation of $S$. We break up the induction in two stages:

$$
\omega=\operatorname{Ind}_{Q}^{G} \operatorname{Ind}_{S}^{Q} 1_{S} .
$$

Let

$$
G^{\prime}=\left\{\left[\begin{array}{ll}
1 & 0 \\
0 & x
\end{array}\right], x \in F^{\times}\right\} \simeq F^{\times} .
$$

Then it is easy to see that

$$
\operatorname{Ind}_{S}^{Q} 1_{S}=1_{S} \otimes \operatorname{regular}_{G^{\prime}} .
$$

The regular representation of $G^{\prime}$ breaks up as a direct integral over all characters. Since induction commutes with direct integrals, we have

$$
\omega=\int_{\widehat{F}^{\times}}^{\oplus} \operatorname{Ind}_{Q^{G}}^{G}\left(1_{S} \otimes \chi\right) d \chi .
$$

To prove (a) it will be enough to show that $\operatorname{Ind}_{Q}^{G} 1_{S} \otimes \chi$ is irreducible when restricted to $Q$ (this will also give the spectral decomposition of $\omega$ ) and that the $\operatorname{Ind}_{Q}^{G} 1_{S} \otimes \chi$ are distinct when restricted to $Q$. 
Let $w=\left[\begin{array}{ll}0 & 1 \\ 1 & 0\end{array}\right]$. Then the double coset $Q w Q$ has total measure in $Q \backslash G / Q$. Note that $Q^{w} \cap Q=A$.

The Mackey decomposition theorem [M2] says that

$$
\left.\left(\operatorname{Ind}_{Q}^{G} 1_{S} \otimes \chi\right)\right|_{Q}=\left.\operatorname{Ind}_{A}^{Q}\left(1_{S} \otimes \chi\right)^{w}\right|_{A} .
$$

We have

$$
\left.\left(1_{S} \otimes \chi\right)^{w}\right|_{A}=\chi \otimes 1_{G^{\prime}},
$$

and it is clear (for example from Mackey decomposition again) that $\left(\operatorname{Ind}_{A}^{Q} \chi \otimes\right.$ $\left.1_{G^{\prime}}\right)\left.\right|_{N}$ is equivalent to the regular representation of $N$. Hence $\operatorname{Ind}_{Q}^{G} \chi \otimes 1_{G^{\prime}}$ is irreducible. Finally

$$
\left(\operatorname{Ind}_{A}^{Q} \chi \otimes 1_{G^{\prime}}\right)\left[\begin{array}{cc}
x & 0 \\
0 & x
\end{array}\right]=\chi(x) I
$$

so that already the $\left.\left(\operatorname{Ind}_{A}^{Q} \chi \otimes 1_{G^{\prime}}\right)\right|_{Z}$ are distinct. Statement (a) is proved.

As for (b), as remarked earlier, (8) gives the spectral decomposition of $\omega$. If $\lambda$ is a character of $G$, then $\lambda$ is of the form $\tilde{\lambda} \circ$ det for some $\tilde{\lambda} \in \widehat{F}^{\times}$. It is then clear that

$$
\lambda \otimes \operatorname{Ind}_{Q}^{G} 1_{S} \otimes \chi=\operatorname{Ind}_{Q}^{G} \tilde{\lambda} \otimes \tilde{\lambda} \chi .
$$

It is well known that if $\alpha, \beta, \gamma, \delta \in \widehat{F}^{\times}$, then $\operatorname{Ind}_{Q}^{G} \alpha \otimes \beta \simeq \operatorname{Ind}_{Q}^{G} \gamma \otimes \delta$ if and only if $\alpha=\gamma$ and $\beta=\delta$ or $\alpha=\delta$ and $\beta=\gamma$ [W, BZ].

Hence the intersection of the spectra of $\lambda \otimes \delta$ and $\lambda^{\prime} \otimes \omega$ consists of only one point and Lemma 1.2 is proved.

We now pass to the proof of Theorem 1.1. We have to show that any $Q_{l}$ morphism of $\rho$ and $\rho^{\prime}$ also intertwines the $G_{n}$-action. If $k=0, \rho$ and $\rho^{\prime}$ are characters (Lemma I.3.5) and the conclusion of the theorem is obvious, since characters of $G_{n}$ are all distinct on $Q_{l}$ for any $l$.

From now on, suppose $k>0$.

We shall use the notation developed in the proof of Theorem I.3.6. We shall also write

$$
\begin{gathered}
\tau=\operatorname{Ind}_{J}^{Q} \omega_{1}, \\
A=\left\{g \in J, g_{i j}=\delta_{i j} \text { if }(i, j) \neq(1,1) \text { or }(n, n)\right\} .
\end{gathered}
$$

The proof of Theorem I.3.6 shows that under our assumptions on the rank of $\rho$,

$$
\left.\rho\right|_{Q_{1}}=\tau \otimes \int_{\widehat{F}^{\times}}^{\oplus} \chi \otimes \nu_{\chi} d \mu(\chi),
$$

where the $\chi$ 's are considered as characters of $A, \nu_{\chi} \in \operatorname{Rep} G_{n-2}$, , and

$$
\operatorname{rk} \nu_{\chi}=\operatorname{rk} \rho-1 \text {. }
$$


We can also write

$$
\left.\rho^{\prime}\right|_{Q_{1}}=\tau \otimes \int_{\widehat{F}^{\times}}^{\oplus} \chi \otimes \nu_{\chi}^{\prime} d \mu^{\prime}(\chi)
$$

with the analogous stipulations.

Suppose first $k=l=1$. This forces $n \geq 4$. Also $\mathrm{rk} \nu_{\chi}=0$ so that $\nu_{\chi}$ is a direct integral of characters. Hence we can write

$$
\left.\rho\right|_{Q_{1}}=\tau \otimes \int_{\left(\widehat{G}_{n-2}\right)_{0} \otimes \hat{F}^{\otimes}}^{\oplus} n_{\lambda, \chi} \lambda \otimes \chi d \tilde{\mu}(\lambda, \chi) .
$$

I claim that $\tilde{\mu}$ is actually concentrated on a single line $\lambda=$ constant. Indeed, write the representation space $\mathscr{H}$ of $\rho$ as

$$
\mathscr{H}=\int_{\left(\widehat{G}_{n-2}\right)_{0}}^{\oplus} \mathscr{H}_{\lambda} d \tilde{\tilde{\mu}}(\lambda)
$$

corresponding to the decomposition of $\left(\widehat{G}_{n-2}\right)_{0} \times F^{\times}$along the lines $\lambda=$ constant. This decomposition is $Q_{1}$-invariant.

Let

(20) $\widetilde{G}_{2}=\left\{g \in G_{n}, g_{i j}=\delta_{i j}\right.$ unless $\left.(i, j)=(1,1),(1, n),(n, 1),(n, n)\right\}$.

Note that $\widetilde{G}_{2}$ commutes with $G_{n-2}$. We claim that the $G_{n-2}$-spectra of the representations $\rho_{\lambda}$ on the $\mathscr{H}_{\lambda}$ are disjoint (apart from sets of zero measure). This will be proved below.

Hence the decomposition (19) is also $\widetilde{G}_{2}$-invariant. Since $\widetilde{G}_{2}$ and $Q_{1}$ generate $G_{n}$, the decomposition is $G_{n}$-invariant. But we are supposing $\rho$ irreducible. Hence $\mathscr{H}=\mathscr{H}_{\lambda}$ for some $\lambda$ and $\tilde{\mu}$ is concentrated on one line.

It is easy to see, for example from the Mackey decomposition theorem [M2], that, since we can choose representatives for $J \backslash Q_{1}$ that centralize $G_{n-2}$,

$$
\left.\tau\right|_{G_{n-2}}=\infty \omega_{1}
$$

Hence

$$
\left.\rho\right|_{G_{n-2}}=\infty \lambda \otimes \omega_{1}
$$

with $\lambda \in\left(\widehat{G}_{n-2}\right)_{0}$. Analogously,

$$
\left.\rho^{\prime}\right|_{G_{n-2}}=\infty \lambda^{\prime} \otimes \omega_{1} .
$$

If $\lambda \neq \lambda^{\prime}$, by our previous claim that the $G_{n-2}$-spectra of $\lambda \otimes \omega_{1}$ and $\lambda^{\prime} \otimes \omega_{1}$ are disjoint, $\operatorname{Hom}_{G_{n-2}}\left(\rho, \rho^{\prime}\right)=\{0\}$. Hence $\operatorname{Hom}_{Q_{1}}\left(\rho, \rho^{\prime}\right)=\{0\}$ and there is nothing to prove. So from now on, suppose that $\lambda=\lambda^{\prime}$. Let

$$
Q_{1}^{(2)}=Q_{1}^{(n)} \cap \widetilde{G}_{2}
$$

Since $\widetilde{G}_{2}$ and $Q_{1}^{(n)}$ generate $Q_{n}$, it will be enough to show that

$$
\operatorname{Hom}_{Q_{1}^{(n)}}\left(\rho, \rho^{\prime}\right) \subseteq \operatorname{Hom}_{\widetilde{G}_{2}}\left(\rho, \rho^{\prime}\right),
$$

or even $\operatorname{Hom}_{Q_{1}^{(2)}}\left(\rho, \rho^{\prime}\right) \subseteq \operatorname{Hom}_{\widetilde{G}_{2}}\left(\rho, \rho^{\prime}\right)$. 
Since $n \geq 4$ we can conjugate $\widetilde{G}_{2}$ into a subgroup of $G_{n-2}$ by an element of $G_{n}$. Let $\omega_{1}^{(2)}$ be as defined in (1). Then it is clear from (I.21) that

$$
\left.\omega_{1}\right|_{\widetilde{G}_{2}}=\infty \omega_{1}^{(2)} .
$$

Also, $\lambda \in\left(\widehat{G}_{n-2}\right)_{0}$ is of the form $\tilde{\lambda} \circ$ det for some $\tilde{\lambda} \in \widehat{F}^{\times}$. Then $\left.\lambda\right|_{\widetilde{G}_{2}}=\tilde{\lambda} \circ \mathrm{det}$ for the same $\tilde{\lambda}$. We conclude that $\left.\rho\right|_{\widetilde{G}_{2}}=\infty \lambda \otimes \omega_{1}^{(2)}=\left.\rho^{\prime}\right|_{\widetilde{G}_{2}}$.

Now Lemma 1.2(a) implies $\operatorname{Hom}_{Q_{1}^{(2)}}\left(\rho, \rho^{\prime}\right)=\operatorname{Hom}_{\widetilde{G}_{2}}\left(\rho, \rho^{\prime}\right)$, which is what we had to show.

To conclude the proof of the case $k=l=1$ we must verify our claim that the $G_{n-2}$-spectra of the $\rho_{\lambda}$ are distinct.

We know that $\left.\rho_{\lambda}\right|_{G_{n-2}}=\infty \lambda \otimes \omega_{1}$ (equation (22)). Since the spectra of $\left.\infty \lambda \otimes \omega_{1}\right|_{\widetilde{G}_{2}}$ and $\left.\infty \lambda \otimes \omega_{1}\right|_{\widetilde{G}_{2}}$ are already disjoint $((25)$ and Lemma 1.2(b)), the claim is true.

Finally, we prove by induction the general case. Recall that we had the decomposition (15) with $\operatorname{rk} \nu_{\chi}=\operatorname{rk} \rho-1$. By induction on $n$ we can assume that

$$
\operatorname{Hom}_{Q_{l-1}^{(n-2)}}\left(\nu_{\chi}, \nu_{\chi}^{\prime}\right)=\operatorname{Hom}_{G_{n-2}}\left(\nu_{\chi}, \nu_{\chi}^{\prime}\right)
$$

Consider the group $M=\left(F^{\times} \times F^{\times}\right) H_{1}$. It acts on $\widehat{H}_{1}$ by conjugation and we have

$$
\operatorname{stab}_{M}\left(\rho_{1}\right)=J \cap M .
$$

The representation $\rho_{1}$ can be extended to $J \cap M$ via the restriction to $J \cap M$ of the representation $\omega_{1}$ of $J$.

Theorem 8.4 of [R] implies that for $\chi \in \widehat{A}, \operatorname{Ind}_{J \cap M}^{M} \chi \otimes \omega_{1}=\chi \otimes \operatorname{Ind}_{J \cap M}^{M} \omega_{1}$ is irreducible. Observe that since $J \cap M \backslash M=J \backslash Q_{1}^{(n)}$, we have $\operatorname{Ind}_{J \cap M}^{M} \omega_{1}=$ $\left.\left(\right.$ Ind $\left._{J}^{Q_{1}} \omega_{1}\right)\right|_{M}=\left.\tau\right|_{M}$. We can obviously extend $\left.\chi \otimes \tau\right|_{M}$ to the representation $\chi \otimes \tau$ of $Q_{1}^{(n)}$. Since $M$ is normal in $Q_{1}^{(n)}$, Theorem 8.4 of [R] says that $\tau \otimes \chi \otimes \otimes_{-}$is an equivalence of categories between the category of representations of $Q_{1}^{(n)}$ which restrict to a multiple of $\left.\chi \otimes \tau\right|_{M}$ and $\operatorname{Rep}\left(Q_{1}^{(n)} / M\right)=\operatorname{Rep} G_{n-2}$. In other words

$$
\operatorname{Hom}_{Q_{1}}\left(\tau \otimes \chi \otimes \nu_{\chi}, \tau \otimes \chi \otimes \nu_{\chi}^{\prime}\right)=\operatorname{Hom}_{G_{n-2}}\left(\nu_{\chi}, \nu_{\chi}^{\prime}\right) .
$$

For the same reason,

$$
\operatorname{Hom}_{Q_{1} \cap Q_{l}}\left(\tau \otimes \chi \otimes \nu_{\chi}^{\prime}, \tau \otimes \chi \otimes \nu_{\chi}^{\prime}\right)=\operatorname{Hom}_{Q_{l-1}^{(n-2)}}\left(\nu_{\chi}, \nu_{\chi}^{\prime}\right)
$$

Hence by (26)

$$
\operatorname{Hom}_{Q_{1} \cap Q_{1}}\left(\rho, \rho^{\prime}\right)=\operatorname{Hom}_{Q_{1}}\left(\rho, \rho^{\prime}\right)
$$


Let $T \in \operatorname{Hom}_{Q_{l}}\left(\rho, \rho^{\prime}\right) \subseteq \operatorname{Hom}_{Q_{l} \cap Q_{1}}\left(\rho, \rho^{\prime}\right)$. Then $T \in \operatorname{Hom}_{Q_{1}}\left(\rho, \rho^{\prime}\right)$ so that $T \in \operatorname{Hom}_{Q_{1}}\left(\rho, \rho^{\prime}\right) \cap \operatorname{Hom}_{Q_{l}}\left(\rho, \rho^{\prime}\right)$. Since $Q_{1}$ and $Q_{l}$ generate $G_{n}, T \in$ $\operatorname{Hom}_{G_{n}}\left(\rho, \rho^{\prime}\right)$. This concludes the proof of Theorem 2.1 .

\section{THE MAP $\theta$}

We have seen that restriction defines an injection $\left(\widehat{G}_{n}\right)_{k} \rightarrow \widehat{G}_{l}^{(n)}$ if $l \geq k$. In fact much more is true, as we shall see in this section and the next.

Let $\rho \in\left(\widehat{G}_{n}\right)_{k}$ and $k<[n / 2]$. Then $\left.\rho\right|_{Q_{k}}$ is still irreducible (Theorem 2.1).

We examine the representation theory of $Q_{k}$. The center $Z$ of the unipotent radical $H_{k}$ of $Q_{k}$ is isomorphic to $\mathscr{M}_{k}$, the additive group of $k \times k$ matrices. Fix a nontrivial character $\chi_{1}$ of $F$. Any character of $\mathscr{M}_{k}$ is of the form $\chi_{1} \circ \lambda$ for some $\lambda \in \mathscr{M}_{k}^{*}$. We identify $\mathscr{M}_{k}^{*}$ with $\mathscr{M}_{k}$ by setting, for $A, B \in \mathscr{M}_{k}$,

$$
\lambda_{A}(B)=\operatorname{trace}(A B) .
$$

The action of $Q_{k}$ on $\widehat{\mathscr{M}}_{k}$ induced by conjugation becomes, under these identifications, the usual action of $G_{k} \times G_{k}$ on $\mathscr{M}_{k}$ by pre- and post-multiplication.

Hence orbits are, for $0 \leq l \leq k$,

$$
\mathscr{O}_{l}^{\prime}=\left\{\lambda_{A}, \operatorname{rank}(A)=l\right\} .
$$

If $\lambda \in \mathscr{M}_{k}^{*}, \operatorname{ker} \lambda$ is a normal subgroup of $H_{k}^{(n)}$. If $\lambda$ has rank $k, H_{k} / \operatorname{ker} \lambda$ is a Heisenberg group with center $\mathscr{M}_{k} / \operatorname{ker} \lambda$. Let $\sigma \in \widehat{H}_{k}$. Then $\left.\sigma\right|_{Z}$ is a multiple of a character. If this character happens to correspond to a functional $\lambda$ of rank $k, \sigma$ factors to $H_{k} / \operatorname{ker} \lambda$. The Stone-Von Neumann theorem [VN] says that there is a unique irreducible representation of $H_{k} / \operatorname{ker} \lambda$ with any fixed nontrivial central character. Hence, for any $\lambda \in \mathscr{O}_{k}^{\prime}$, there is a unique irreducible representation $\rho_{\lambda}$ of $H_{k}$ which restricts to $\chi_{1} \circ \lambda$ on $Z$.

If we write

$$
H_{k}=\left\{(x, y, z), x \in \mathscr{M}_{k, n-2 k}, y \in \mathscr{M}_{n-2 k, k}, \quad z \in \mathscr{M}_{k}\right\}
$$

corresponding to the block decomposition

$$
\left[\begin{array}{lll}
I & x & z \\
0 & I & y \\
0 & 0 & I
\end{array}\right],
$$

and we set $X=\{(x, 0,0)\}$, then $\rho_{\lambda}$ is realized on $L^{2}(X)$ by

$$
\begin{aligned}
& \left(\rho_{\lambda}(x, 0,0) f\right)\left(x^{\prime}\right)=f\left(x+x^{\prime}\right), \\
& \left(\rho_{\lambda}(0, y, 0) f\right)\left(x^{\prime}\right)=\chi_{1}\left(\lambda\left(x^{\prime} y\right)\right) f\left(x^{\prime}\right), \\
& \left(\rho_{\lambda}(0,0, z) f\right)\left(x^{\prime}\right)=\chi_{1}(\lambda(z)) f\left(x^{\prime}\right) .
\end{aligned}
$$

Let $i: \mathscr{M}_{k} \rightarrow N_{k}$ be the natural injection of abelian groups. If $\rho \in \operatorname{Rep} N_{k^{\prime}}$ the $\mathscr{M}_{k}$-spectral measure of $\rho$ is the image of the $N_{k}$-spectral measure of $\rho$ under 
the adjoint projection $i^{*}: N_{k}^{*} \rightarrow \mathscr{M}_{k}^{*}$. If $\rho \in\left(\widehat{G}_{n}\right)_{k}$, its $N_{k}$-spectral measure is Lebesgue measure on the orbit $\mathscr{O}_{k} \subseteq N_{k}^{*}$.

Hence $\rho$ obviously has $\mathscr{M}_{k}$-spectral measure concentrated on $\mathscr{O}_{k}^{\prime}$. The trace functional on $\mathscr{M}_{k}$ has rank $k$. Denote it by tr. $Q_{k}$ acts on $\widehat{H}_{k}$ by conjugation. Note that

$$
\begin{aligned}
\operatorname{stab}_{Q_{k}}\left(\rho_{\mathrm{tr}}\right) & =\operatorname{stab}_{Q_{k}}(\operatorname{tr}) \\
& =J=\left\{g \in Q_{k}, g_{i j}=g_{i+n-k, j+n-k} \text { for all } 1 \leq i, j \leq k\right\} .
\end{aligned}
$$

Let

$$
\widehat{J}_{\mathrm{tr}}=\left\{\tau \in \widehat{J},\left.\tau\right|_{H_{k}} \text { is a multiple of } \rho_{\mathrm{tr}}\right\}
$$

Theorem 1.1 of [R] says that $\operatorname{Ind}_{J}^{Q_{k}}$ is an equivalence of categories between the category of representations of $Q_{k}$ which live on $\mathscr{O}_{k}^{\prime}$ and the category of representations of $J$ which restrict to a multiple of $\rho_{\mathrm{tr}}$ on $H_{k}$.

Since $\left.\rho\right|_{Q_{k}}$ is irreducible, $\left.\rho\right|_{Q_{k}}=$ Ind $_{j}^{Q_{k}} \tau$ for some $\tau \in \widehat{J}_{\mathrm{tr}}$.

One can easily check that $\rho_{\mathrm{tr}}$ extends to $J$ in the following way. The Levi component of $J$ is the group

$$
M=\left\{\left[\begin{array}{ccc}
A & 0 & 0 \\
0 & B & 0 \\
0 & 0 & A
\end{array}\right], A \in G_{k}, B \in G_{n-2 k}\right\} .
$$

The representation $\omega_{\mathrm{tr}}$ of $M$ acts on $L^{2}(X)$ and is given by

$$
\left(\omega_{\operatorname{tr}}\left[\begin{array}{ccc}
A & 0 & 0 \\
0 & B & 0 \\
0 & 0 & A
\end{array}\right] f\right)\left(x^{\prime}\right)=|\operatorname{det} A|^{(2 k-n) / 2}|\operatorname{det} B|^{k / 2} f\left(A^{-1} x^{\prime} B\right) \text {. }
$$

We shall denote by $\omega_{\mathrm{tr}}$ the representation of $J$ which is $\rho_{\mathrm{tr}}$ on $H_{k}$ and $\omega_{\mathrm{tr}}$ on $M$.

Again [R, Theorem 8.4] tells us that $\left.\rho\right|_{Q_{k}}=\operatorname{Ind}_{J}^{Q_{k}} \omega_{\mathrm{tr}} \otimes \nu$ with $\nu \in \widehat{M}=$ $\left(G_{k} \times G_{n-2}\right)^{\wedge}$.

Hence $\nu$ is of the form $\nu=\sigma \otimes \nu^{\prime}$ with $\sigma \in \widehat{G}_{k}, \nu^{\prime} \in \widehat{G}_{n-2 k}$. The same argument as in the proof of Theorem I.3.6 now shows that

$$
\mathrm{rk} \nu^{\prime}=\mathrm{rk} \rho-k=0
$$

so that $\nu^{\prime}$ is actually a character, hence of the form $\chi \circ \operatorname{det}$ for some $\chi \in \widehat{F}^{\times}$. Thus if $\rho \in\left(\widehat{G}_{n}\right)_{k}$,

$$
\left.\rho\right|_{Q_{k}}=\operatorname{Ind}_{J}^{Q_{k}} \omega_{\mathrm{tr}} \otimes \sigma \otimes \chi \circ \mathrm{det} .
$$

Theorem 2.1 says that $\left.\rho \mapsto \rho\right|_{Q_{k}}$ is an injection and Mackey theory says that $\left.\rho\right|_{Q_{k}} \mapsto(\sigma, \chi)$ is an injection.

We summarize the discussion as follows: 
Theorem 2.1. Let $k<[n / 2]$. There is an injective map $\theta:\left(\widehat{G}_{n}\right)_{k} \rightarrow \widehat{G}_{k} \times \widehat{F}^{\times}$ such that if $\theta(\rho)=(\sigma, \chi),\left.\rho\right|_{Q_{k}}=\operatorname{Ind}_{J}^{Q_{k}} \omega_{\mathrm{tr}} \otimes \sigma \otimes \chi \circ \mathrm{det}$.

\section{Classification of SMAll Representations}

We shall now show that the map $\theta$ constructed above is a surjection and give an explicit construction for the inverse of $\theta$. This will give a classification of representations of small rank.

As a byproduct, we shall obtain the irreducibility of certain induced representations of $G_{n}$.

To simplify notation, whenever $\chi \in \widehat{F}^{\times}$, we shall denote the character $\chi$ odet of $G_{n}$ simply by $\chi$. If $\chi$ is a character of $G_{n}$ and $\rho \in \operatorname{Rep} G_{n}$, we denote by $\chi \rho$ the representation $\chi \otimes \rho$ of $G_{n}$. Finally if $\rho \in \operatorname{Rep} G_{k}$ and $\sigma \in \operatorname{Rep} G_{l}$, we denote by $\rho \times \sigma$ the representation of $G_{k+l}$ obtained by extending the representation $\rho \otimes \sigma$ of $G_{k} \times G_{l}$ trivially to $P_{k}^{(k+l)}$ and then inducing up to $G_{k+l}$.

With this notation we have

Theorem 3.1. Let $k<[n / 2], \sigma \in \widehat{G}_{k}, \chi \in \widehat{F}^{\times}$. Consider $\chi$ as a character of $G_{n-k}$. Then $\chi^{-1} \sigma \times \chi \in\left(\widehat{G}_{n}\right)_{k}$ and the maps $\theta$ and $(\sigma, \chi) \rightarrow \chi^{-1} \sigma \times \chi$ are inverses.

Corollary 3.2. $\left(\widehat{G}_{n}\right)_{k}=\left\{\tau \times \chi, \tau \in \widehat{G}_{k}, \chi \in\left(\widehat{G}_{n-k}\right)_{0}\right\}$.

Corollary 3.3. The representations $\tau \times \chi$ of $G_{k+l}$, with $\tau \in \widehat{G}_{k}$ and $\chi \in\left(\widehat{G}_{l}\right)_{0}$, are irreducible whenever $l \geq k$.

Note. A slightly more general result than Corollary 3.3 was proved, by completely different methods, by S. Sahi [Sa1]. The irreducibility of all representations of the form $\tau \times \sigma$, with $\tau, \sigma$, unitary would follow from Kirillov's conjecture that all irreducible unitary representations of $G_{n}$ stay irreducible when restricted to $P_{1}^{(n)}$. The conjecture has been shown to be true for $F$ nonArchimedean by J. Bernstein [B] and for $F=\mathbf{C}$ by S. Sahi [Sa2].

Proof of Theorem 3.1. Because of Theorems 1.1 and 2.1 it is sufficient to show:

(1) $\chi^{-1} \sigma \times \chi$ has pure rank $k$.

(2) $\chi^{-1} \sigma \times\left.\chi\right|_{Q_{k}} \simeq \operatorname{Ind}_{J}^{Q_{k}} \omega_{\mathrm{tr}} \otimes \sigma \otimes \chi$.

In fact, it suffices to prove (2) because Ind $_{J}^{Q_{k}} \omega_{\mathrm{tr}} \otimes \sigma \otimes \chi$ has pure $N_{l}$-rank $k$ if $k<l \leq[n / 2]$. This follows from equation (40) in $\S 3$. Statement (1) can also be proved directly by means of the Mackey decomposition theorem [M2]. We omit the details.

We begin the proof of (2) by applying the Mackey decomposition theorem to the groups $G_{n}, P_{k}, Q_{k}$.

There is one double coset of total measure in $P_{k} \backslash G_{n} / Q_{k}$, a coset representative of which is the element $w$ of $G_{n}$ given by

$$
w_{i j}=\delta_{i, n+1-j} \text {. }
$$


We denote by $M$ the group

$$
M=P_{k}^{w} \cap Q_{k}=\left\{\left[\begin{array}{lll}
* & * & 0 \\
0 & * & 0 \\
0 & 0 & *
\end{array}\right]\right\} .
$$

Here $*$ denotes any matrix of the right size, subject to the stipulation that the element of $\mathscr{M}_{n}$ which we obtain is invertible.

The representation $\left(\chi^{-1} \sigma \otimes \chi\right)^{w}$ is a representation of $M$ as in the diagram

$$
\left[\begin{array}{lll}
\chi & \chi & \\
& \chi & \\
& & \chi^{-1} \sigma
\end{array}\right]
$$

Hence we must prove

$$
\operatorname{Ind}_{M}^{Q_{k}}\left(\chi \otimes \chi^{-1} \sigma\right) \simeq \operatorname{Ind}_{J}^{Q_{k}} \omega_{\mathrm{tr}} \otimes \sigma \otimes \chi .
$$

We shall do this by finding explicitly an intertwining operator between the two representations.

Note that

$$
Q_{k}=M \ltimes N_{n-k} .
$$

In the rest of the proof we shall denote $Q_{k}$ by $Q$ and $N_{n-k}$ by $N$. Also denote $\chi \otimes \chi^{-1} \sigma \in \operatorname{Rep} M$ by $\bar{\sigma}$ and $\operatorname{Ind}_{M}^{Q} \bar{\sigma}$ by $\pi$.

It is clear that restriction to $N$ defines a unitary bijection between the representation space $\mathscr{H}_{\pi}$ of $\pi$ and $L^{2}\left(N ; \mathscr{H}_{\sigma}\right)$. Elementary computations show that in this realization of $\pi$ on $L^{2}\left(N ; \mathscr{H}_{\sigma}\right)$

$$
(\pi(m u) f)\left(u_{0}\right)=\bar{\sigma}(m) f\left(\left(u_{0} \cdot m\right) u\right) \Delta_{N}(m)^{1 / 2} .
$$

Here $u_{0} \cdot m=m^{-1} u m$ and $\Delta_{N}$ denotes the modular function for the action of $M$ on $N$.

Let now $\mathscr{F}: L^{2}\left(N ; \mathscr{H}_{\sigma}\right) \rightarrow L^{2}\left(N^{*} ; \mathscr{H}_{\sigma}\right)$ be given by Fourier transform. In other words

$$
(\mathscr{F} f)(v)=\int_{N} \chi_{1}(\langle u, v\rangle) f(u) d u
$$

then $\pi^{\prime}=\mathscr{F} \pi \mathscr{F}^{-1}$ is equivalent to $\pi$ and

$$
\left(\pi^{\prime}(m u) f\right)(v)=\Delta_{N}(m)^{1 / 2} \bar{\sigma}(m) \chi_{1}\left(\left\langle u^{-1} \cdot m^{-1}, v\right\rangle\right) f\left(v \cdot{ }^{t} m^{-1}\right) .
$$

Here $v \cdot{ }^{t} m$ denotes the transpose action of $M$ on $N^{*}$.

Let us now turn to the other representation involved. Let $\zeta=\omega_{\mathrm{tr}} \otimes \sigma \otimes \chi \in \hat{J}$ and $\nu=\operatorname{Ind}_{J}^{Q} \zeta$.

The representation space $\mathscr{H}_{\nu}$ of $\nu$ is the space of functions $f: Q \rightarrow \mathscr{H}_{\zeta}$ which satisfy

(1) for all $j \in J$ and $q \in Q, f(j q)=\zeta(j) f(q)$;

(2) the function $q \mapsto\|f(q)\|$ is in $L^{2}(J \backslash Q, d \mu)$, 
where $\mu$ is some quasi-invariant measure on $J \backslash Q$. Set

$$
\lambda\left(q_{0}, q\right)=(d(\mu \cdot q) / d \mu)\left(J q_{0}\right) .
$$

Then the action of $Q$ on $\mathscr{H}_{\nu}$ is given by

$$
(\nu(q) f)\left(q_{0}\right)=\lambda\left(q_{0}, q\right)^{1 / 2} f\left(q_{0} q\right) .
$$

We fix $\mu$ as follows: If

$$
q=\left[\begin{array}{lll}
W & X & R \\
0 & Y & S \\
0 & 0 & Z
\end{array}\right]
$$

set

$$
s(J q)=\left[\begin{array}{ccc}
Z W^{-1} & 0 & 0 \\
0 & I & 0 \\
0 & 0 & I
\end{array}\right]
$$

We take $\mu$ to be the preimage under $s$ of Lebesgue measure on $\mathscr{M}_{k}$.

It is easy to see that if $q$ is given by $(51)$, then

$$
\lambda\left(q_{0}, q\right)=|\operatorname{det} Z|^{-k}|\operatorname{det} W|^{k} .
$$

We shall denote $\lambda\left(q_{0}, q\right)$ by $\Delta^{\prime}(q)$. Let $U^{\prime}: \mathscr{H}_{\nu} \rightarrow L^{2}\left(J \backslash Q, d \mu ; \mathscr{H}_{\zeta}\right)$ be given by restriction to $s(J \backslash Q)$ followed by $s^{*}$. $U^{\prime}$ is a unitary bijection, so that if $\nu^{\prime}=U^{\prime} \nu U^{\prime-1}, \nu^{\prime}$ is equivalent to $\nu$. If $\bar{q}_{0}=J q_{0}$, define

$$
j\left(\bar{q}_{0}, q_{0}\right)=s\left(\bar{q}_{0}\right) q s\left(\overline{q_{0} q}\right)^{-1} .
$$

Then it is easy to compute

$$
\nu^{\prime}(q) f\left(\bar{q}_{0}\right)=\Delta^{\prime}(q)^{1 / 2} \zeta\left(j\left(\bar{q}_{0}, q\right)\right) f\left(\overline{q_{0} q}\right) .
$$

Let

$$
\bar{q}_{0}=\left[\begin{array}{lll}
A & 0 & 0 \\
0 & I & 0 \\
0 & 0 & I
\end{array}\right]
$$

Then

$$
j\left(\bar{q}_{0}, m\right)=\left[\begin{array}{ccc}
Z & A X & 0 \\
0 & Y & 0 \\
0 & 0 & Z
\end{array}\right] \quad \text { if } m=\left[\begin{array}{ccc}
W & X & 0 \\
0 & Y & 0 \\
0 & 0 & I
\end{array}\right] \text {, }
$$

and

$$
j\left(\bar{q}_{0}, u\right)=\left[\begin{array}{ccc}
I & 0 & A R \\
0 & I & S \\
0 & 0 & I
\end{array}\right] \quad \text { if } u=\left[\begin{array}{ccc}
I & 0 & R \\
0 & I & S \\
0 & 0 & I
\end{array}\right] .
$$

We have $\mathscr{H}_{\nu}^{\prime}=L^{2}\left(\mathscr{M}_{k}, \mathscr{H}_{\sigma} \otimes L^{2}(X)\right)$ and $\mathscr{H}_{\pi^{\prime}}=L^{2}\left(\mathscr{M}_{k}^{*} \times S^{*}, \mathscr{H}_{\sigma}\right)$, where

$$
X=\left\{\left[\begin{array}{ccc}
I & X & 0 \\
0 & I & 0 \\
0 & 0 & I
\end{array}\right]\right\}
$$


and

$$
S=\left\{\left[\begin{array}{lll}
I & 0 & 0 \\
0 & I & S \\
0 & 0 & I
\end{array}\right]\right\}
$$

We identify $\mathscr{M}_{k}^{*} \times S^{*}$ with $\mathscr{M}_{k} \times X$ via

$$
\left\langle(A, \xi),\left[\begin{array}{c}
R \\
\eta
\end{array}\right]\right\rangle=\operatorname{tr}(A R+\xi \eta),
$$

where $A \in \mathscr{M}_{k}, \xi \in X, R \in \mathscr{M}_{k}, \eta \in S$.

The Hilbert spaces $\mathscr{H}_{\nu^{\prime}}$ and $\mathscr{H}_{\pi^{\prime}}$ are canonically isomorphic. Indeed linear combinations of vectors of the form $F(A)=f(A) g \otimes v$ with $f \in L^{2}\left(\mathscr{M}_{k}\right)$, $g \in L^{2}(X), v \in \mathscr{H}_{\sigma}$ are dense in $\mathscr{H}_{\nu^{\prime}}$.

Analogously linear combinations of vectors of the form $g(A, \xi)=f(A) g(\xi) v$ with $f \in L^{2}\left(\mathscr{M}_{k}^{*}\right), g \in L^{2}\left(S^{*}\right)$ and $v \in \mathscr{H}_{\sigma}$ are dense in $\mathscr{H}_{\pi^{\prime}}$.

The canonical isomorphism consists of identifying $F(A)$ and $G(A, \xi)$, using identification (58).

Let $m, u$ be as above. Then we calculate

$\left(\nu^{\prime}(m) F\right)(A)=|\operatorname{det} Z|^{k / 2}|\operatorname{det} W|^{k / 2} \chi(Y)\left(\omega_{\mathrm{tr}}(Z, Y, A X) g\right) \otimes \sigma(Z) v f\left(Z^{-1} A W\right)$ and

$$
\left(\nu^{\prime}(u) F\right)(A)=\omega_{\mathrm{tr}}(A R, S) \otimes v f(A) .
$$

We then wish to calculate the transpose action of $M$ on $\mathscr{M}_{k}^{*} \times S^{*}$ under our identification. We obtain

$$
(A, \xi) \cdot{ }^{t} m=\left(Z A W^{-1},-Z A W^{-1} X Y^{-1}+Z \xi Y^{-1}\right) .
$$

Finally we compute

$$
\begin{aligned}
\pi^{\prime}(m) G(A, \xi)= & |\operatorname{det} W|^{k / 2}|\operatorname{det} Z|^{(n-k) / 2}|\operatorname{det} Y|^{k / 2} \chi(W) \chi(Y) \chi(Z)^{-1} \\
& \cdot \sigma(Z) v f\left(Z^{-1} A W\right) g\left(Z^{-1} A X+Z^{-1} \xi Y\right)
\end{aligned}
$$

and

$$
\left(\pi^{\prime}(u) G\right)(A, \xi)=\chi_{1}(\operatorname{tr}(-A R-\xi S)) G(A, \xi) .
$$

Comparison of (59), (60) with (62), (63) (see also (35), (39)) shows that the two representations are realized by "almost" the same formulae. The only discrepancy is the factor $\chi(W) \chi(Z)^{-1}$ which appears in (63) and not in (60). This can be eliminated easily, however, by use of the unitary operator $T_{\chi}: L^{2}\left(\mathscr{M}_{k}\right) \rightarrow$ $L^{2}\left(\mathscr{M}_{k}\right)$ given by

$$
\left(T_{\chi} f\right)(A)=\chi^{-1}(A) f(A) .
$$

This concludes the proof of Theorem 4.1.

The computations in the proof are carried out in detail in [S]. 


\section{ASYMPTOTICS OF MATRIX COEFFICIENTS}

\section{NotATION AND BASIC NOTIONS}

Let $G$ be a locally compact group with center $Z$. Fix a compact subgroup $K$ of $G$. If $\rho \in \operatorname{Rep} G$ and $u, v$ are in the representation space of $\rho$, denote by $\phi_{u, v}$ the matrix coefficient

$$
\phi_{u, v}(g)=(\rho(g) u, v) .
$$

Definition 1.1. Suppose $\rho \in \operatorname{Rep} G$ with representation space $\mathscr{H}$. Suppose further that $Z$ acts by a character (this is always true if, for example, $\rho$ happens to be irreducible). We say $\rho$ is an $L^{p}(G / Z)$-representation if there exists a dense subspace $\mathscr{U}$ of $\mathscr{H}$ such that $\left|\phi_{u, v}\right| \in L^{p}(G / Z)$ whenever $u, v \in \mathscr{U}$.

Now let $\rho \in \operatorname{Rep} G$ have representation space $\mathscr{H}$. Write

$$
\mathscr{H}=\bigoplus_{\mu \in \hat{K}} \mathscr{H}_{\mu},
$$

where $K$ acts on $\mathscr{H}_{\mu}$ by a multiple of $\mu$.

Definition 1.2. Let $\rho \in \operatorname{Rep} G$ and let $\Phi$ be a nonnegative function on $G$ satisfying

$$
\Phi(g)=\Phi\left(g^{-1}\right), \quad \Phi\left(z k_{1} g k_{2}\right)=\Phi(g)
$$

whenever $g \in G, k_{1}, k_{2} \in K, z \in Z$. Let $\Psi$ be a function on $\hat{K}$. We say $\rho$ is $(\Phi, \Psi)$-bounded if whenever $\mu, \nu \in \hat{K}$ and $u \in \mathscr{H}_{\mu}, \nu \in \mathscr{H}_{\nu}$

$$
\left|\Phi_{u, v}(g)\right| \leq \Phi(g) \Psi(\mu) \Psi(\nu)\|u\|\|v\| .
$$

Proposition 1.3. Suppose $\rho$ is $(\Phi, \Psi)$-bounded and $\Phi \in L^{p}(G / Z)$. Then $\rho$ is an $L^{p}(G / Z)$-representation.

While the definition of $(\Phi, \Psi)$-boundedness is more cumbersome that the definition of $L^{p}$-ness of representations, it is easier to work with for our purposes. We give two lemmas which will be useful later.

Lemma 1.4. Let $\rho=\int_{X}^{\oplus} \sigma_{x} d \mu(x)$. Then $\rho$ is $(\Phi, \Psi)$-bounded if and only if $\sigma_{x}$ is $(\Phi, \Psi)$-bounded for almost all $x$.

Lemma 1.5. The set of $(\Phi, \Psi)$-bounded representations is closed in $\operatorname{Rep} G$ (with respect to the Fell topology).

The proofs are easy and left to the reader.

We now specialize these notions to the case of general linear groups. We shall write $B_{n}=\left\{g \in G_{n}, g_{i j}=0\right.$ if $\left.i>j\right\}$, the Borel subgroup of upper triangular matrices, and $A_{n}=\left\{g \in G_{n}, g_{i j}=0\right.$ if $\left.i \neq j\right\}$, the maximal torus of diagonal matrices.

We shall denote by $K_{n}$ a maximal compact subgroup of $G_{n}$ as follows. If $F=\mathbf{R}, K_{n}$ will be the group $\mathrm{O}(n)$ of orthogonal matrices. If $F=\mathbf{C}, K_{n}$ will 
denote the group $\mathrm{U}(n)$ of unitary matrices. If $F$ is non-Archimedean and $\mathscr{O}$ is its ring of integers, $K_{n}$ shall denote the group $\mathrm{GL}_{n}(\mathscr{O})$ of matrices whose entries lie in $\mathscr{O}$ and whose determinant is invertible in $\mathscr{O}$.

We have the Iwasawa decomposition $G_{n}=B_{n} K_{n}$.

Let $P$ be any subgroup of $G_{n}$ containing $B_{n}$. Let $\sigma \in \operatorname{Rep} P$ be realized on $\mathscr{H}_{\sigma}$. Because of the Iwasawa decomposition, $\operatorname{Ind}_{P}^{G_{n}} \sigma$ can be realized on the space $\mathscr{H}$ of functions $u$ from $K_{n}$ to $\mathscr{H}_{0}$ satisfying

(1) $u$ is measurable,

(2) If $p \in K_{n} \cap P$, then $u(p k)=\sigma(p) u(k)$ for all $k \in K_{n}$.

(3) $\|u(k)\| \in L^{2}\left(K_{n} \cap P \backslash K_{n}, \mu\right)$, where $\mu$ is the unique $K_{n}$-invariant measure on $K_{n} \cap P \backslash K_{n}$.

Let $k: G_{n} \rightarrow K_{n}$ and $b: G_{n} \rightarrow B_{n}$ be measurable functions such that for all $g \in G_{n}$

$$
g=b(g) k(g) .
$$

Then a formula for $\operatorname{Ind}_{p}^{G_{n}} \sigma$ is

$$
\left(\left(\operatorname{Ind}_{P}^{G_{n}} \sigma\right)(g) u\left(k^{\prime}\right)=\lambda\left(k^{\prime}, g\right)^{1 / 2} \sigma\left(b\left(k^{\prime} g\right)\right) u\left(k\left(k^{\prime} g\right)\right),\right.
$$

where

$$
\lambda\left(k^{\prime}, g\right)=\frac{d(\mu \cdot g)}{d \mu}\left(k^{\prime}\right) .
$$

Note that $\lambda\left(k^{\prime}, k\right)=1$ for all $k \in K_{n}$.

It is easy to check that $u \in \mathscr{H}$ is $K_{n}$-invariant if and only if $u(k)=\bar{u}$ for all $k \in K_{n}$ with $\bar{u}$ a $K_{n} \cap P$-invariant vector in $\mathscr{H}_{\sigma}$. Let $U_{n}$ be the unipotent radical of $B_{n}$, i.e. the group

$$
U_{n}=\left\{g \in B_{n}, g_{i i}=1 \text { for all } i\right\} .
$$

The torus $A_{n}$ acts on $U_{n}$ by conjugation. Let $\Sigma^{+}$be the set of positive roots with respect to this action:

$$
\Sigma^{+}=\left\{\alpha: A \rightarrow F^{\times}, \exists u \in U_{n}-\{I\} \text { s.t. } u^{a}=\alpha(a)(u-I)+I\right\} .
$$

The positive Weyl chamber in $A_{n}$, denoted by $A_{n}^{+}$, is

$$
A_{n}^{+}=\left\{a \in A_{n},|\alpha(a)| \geq 1 \text { for all } \alpha \in \Sigma^{+}\right\} .
$$

It is very easy to make the above explicit:

$$
\Sigma^{+}=\left\{\alpha_{i j}, i>j\right\}
$$

where

$$
\alpha_{i j}\left(\operatorname{diag}\left(a_{1}, \ldots, a_{n}\right)\right)=a_{i} a_{j}^{-1}
$$

so that

$$
A_{n}^{+}=\left\{\operatorname{diag}\left(a_{1}, \ldots, a_{n}\right),\left|a_{1}\right| \leq \cdots \leq\left|a_{n}\right|\right\} .
$$


It is easy to check that the Radon-Nikodým derivative of the action of $G_{n}$ on $K_{n} \cap B_{n} \backslash K_{n}$ is given by

$$
\lambda\left(k^{\prime}, g\right)=\delta_{B_{n}}(b(g)),
$$

where $\delta_{B_{n}}$, the modular function of $B_{n}$, is the group morphism $B_{n} \rightarrow \mathbf{R}^{+}$ determined by

$$
\begin{aligned}
& \delta_{B_{n}}(a)=\prod_{\alpha \in \Sigma^{+}}|\alpha(a)| \text { for } a \in A_{n}, \\
& \delta_{B_{n}}(u)=1 \text { for } u \in U_{n} .
\end{aligned}
$$

Let $\tau$ denote the trivial representation of $B_{n}$. Up to multiples, there is a unique $K_{n}$-invariant vector $u$ in $\operatorname{Ind}_{B_{n}}^{G_{n}} \tau$, given by $u(k)=1$. Its matrix coefficient $\Xi_{n}$, called the Harish-Chandra spherical function, is given by

$$
\Xi_{n}(g)=\int_{K_{n}} \delta^{1 / 2}(b(k g)) d k .
$$

Proposition 1.6 [HC, Si]. There exist constant $c_{1}, c_{2}(\varepsilon)$ such that for all $\alpha \in A_{n}^{+}$ and $\varepsilon>0$

$$
c_{1} \delta_{B_{n}}^{-1 / 2}(a) \leq \Xi_{n}(a) \leq c_{2}(\varepsilon) \delta_{B_{n}}^{-1 / 2+\varepsilon}(a) .
$$

We have the Cartan decomposition $G_{n}=K_{n} A_{n}^{+} K_{n}$.

Proposition 1.7. Haar measure on $G_{n}$ with respect to the Cartan decomposition is given by

$$
d g=\Delta(a) d k_{1} d a d k_{2}
$$

where $\Delta(a)$ is a positive function on $A_{n}^{+}$satisfying

$$
d_{1}(t) \delta_{B_{n}}(a) \leq \Delta(a) \leq d_{2} \delta_{B_{n}}(a)
$$

for all $a \in\left\{\alpha \in A_{n}^{+},|\alpha(a)| \geq t\right.$ for all $\left.\alpha \in \Sigma^{+}\right\}$and for some constants $d_{2}$ and $d_{1}(t)$ with $d_{2}(t)>0$ if $t>1$.

For the proof, see e.g. [W].

Corollary 1.8. If $\rho \in \operatorname{Rep} G_{n}$ is $\left(\Xi_{n}^{r}, \Psi\right)$-bounded for some $r>0$ and some $\Psi$, then $\rho$ is a $L^{2 / r+\varepsilon}\left(G_{n} / Z\right)$-representation for all $\varepsilon>0$.

Finally, we state an important result we shall need in the sequel.

Theorem 1.9. The regular representation $\lambda$ of $G_{n}$ (and hence, by Lemma 1.5 any representation in the closure in $\operatorname{Rep} G_{n}$ of $\left.\{\lambda\}\right)$ is $\left(\Xi_{n}, \operatorname{dim} \mu\right)$-bounded.

For the proof, see [H1].

\section{RESTRICTING REPRESENTATIONS}

Definition 2.1. Let $I=\left\{i_{1}, \ldots, i_{k}\right\} \subseteq\{1, \ldots, n\}$ with $i_{1}<i_{2}<\cdots<i_{n}$. The standard embedding $\phi_{I}$ of $G_{k}$ in $G_{n}$ corresponding to $I$ is the injection such 
that the map from $F^{k}$ to $F^{n}$ given by $e_{j} \rightarrow e_{i_{j}}$ for $j=1, \ldots, k$ intertwines the standard action of $G_{k}$ on $F^{k}$ and the action of $G_{k}$ on $F^{n}$ induced by $\phi_{I}$. Theorem 2.2. Suppose $\rho \in \operatorname{Rep} G_{n}$ has pure rank $k \neq n / 2$. Then for any standard embedding of $G_{k+1},\left.\rho\right|_{G_{k+1}}$ is $\left(\Xi_{k+1}, \operatorname{dim} \mu\right)$-bounded. If $k=n / 2$ (in particular $n$ is even $),\left.\rho\right|_{G_{k}}$ is $\left(\Xi_{k}, \operatorname{dim} \mu\right)$-bounded for any standard embedding of $G_{k}$.

Note. It is easy to see from the definition of $\delta_{B_{n}}$ in Proposition 1.6 that $\Xi_{k} \geq$ $\left.\Xi_{k+1}\right|_{G_{k}}$. Hence the result for $k=n / 2$ is weaker than the others.

This does not arise from a defect in the proof, but from an actual feature of representation theory of $G_{n}$, namely the existence of the complementary series.

Let $\chi_{\alpha}$ be the nonunitary character of $P_{k}^{(2 k)}$ which is trivial on $N_{k}^{(2 k)}$ and given by

$$
\chi_{\alpha}=|\operatorname{det}|^{\alpha} \otimes|\operatorname{det}|^{-\alpha}, \quad \alpha \in \mathbf{R},
$$

on the Levi component of $P_{k}^{(2 k)}$ (which is isomorphic to $G_{k} \times G_{k}$ ).

It is well known [St] that $\operatorname{Ind}_{P_{k}}^{G_{2 k}} \chi_{\alpha}$ can be "unitarized" if $-\frac{1}{2}<\alpha<\frac{1}{2}$, giving rise to a family of unitary representations of $G_{2 k}$, called the complementary series.

These representations are easily seen to have rank $k$ (for example this follows from the results of Chapter II).

It is also easy to see that $\left.\operatorname{Ind}_{P_{k}}^{G_{2 k}} \chi_{\alpha}\right|_{G_{k+1}}$ are not $\left(\Xi_{k+1}, \Psi\right)$-bounded for any $\Psi$, thus giving a counterexample to the possible cleaner version of Theorem 2.2.

The rest of this section is dedicated to the proof of Theorem 2.2

First of all, since all the images of standard embeddings of $G_{k+1}$ (respectively $G_{k}$ ) are conjugate, it is clearly enough to consider the case $I=\{1, \ldots, k+1\}$ (respectively $I=\{1, \ldots, k\}$ ).

First suppose $k \neq n / 2$. Consider the subgroup $P_{k+1}^{(n)}$. We want to analyze representations of $P_{k+1}^{(n)}$ of rank $k$ from the point of view of their restriction of $G_{k+1}$.

We identified $\widehat{N}_{k+1}$ with $\operatorname{Hom}_{F}\left(F^{k+1}, F^{n-(k+1)}\right)$. Let

$$
\mathscr{V}=\left\{\text { subspaces of } F^{n-(k+1)} \text { of dimension } k\right\} \text {. }
$$

$G_{k+1}$ acts on $\operatorname{Hom}_{F}\left(F^{k+1}, F^{n-(k+1)}\right)$ by right multiplication. Hence the $G_{k+1}$ $\times G_{n-(k+1)}$-orbit $\mathscr{O}_{k}$ in $\widehat{N}_{k+1}$ decomposes into the $G_{k+1}$-orbits

$$
\mathscr{O}_{V}=\left\{T \in \operatorname{Hom}_{F}\left(F^{k+1}, F^{n-(k+1)}\right), \operatorname{Im} T=V\right\}
$$

as $V$ varies in $\mathscr{V}$. Hence if $\rho \in \operatorname{Rep} P_{k+1}$ has rank $k$,

$$
\left.\rho\right|_{G_{k+1}}=\int_{\mathscr{V}}^{\oplus} \rho_{V} d \mu(V)
$$


The $\rho_{V}$ are all isomorphic. Indeed $G_{n-(k+1)}$ acts transitively on $\mathscr{V}$ and commutes with $G_{k+1}$.

Since $(\Phi, \Psi)$-boundedness is preserved by direct integrals (Lemma 1.4) it will be enough to prove $\left(\Xi_{k+1}\right.$, $\left.\operatorname{dim} \mu\right)$-boundedness for a single $\rho_{V}$. To fix ideas, let

$$
V=\operatorname{span}\left\{e_{k+2}, \ldots, e_{2 k+1}\right\} .
$$

Let $T: e_{i} \mapsto e_{i+k+1}$ for $1 \leq i \leq k$ and $T\left(e_{k+1}\right)=0$. Then $T \in \mathscr{O}_{V}$. Let

$$
S=\operatorname{stab}_{G_{k+1}}(T)=\left\{g \in G_{k+1}, g_{i j}=\delta_{i j} \text { unless } j=k+1\right\} .
$$

Theorem 1.1 of [R] says that any unitary representation of $G_{k+1} N_{k+1}$ whose spectral measure is concentrated on $\mathscr{O}_{V}$ is induced from a representation of $S N_{k+1}$ whose restriction to $N_{k+1}$ is a multiple of the character $\chi_{T}$ corresponding to $T$.

Since $S N_{k+1}$ is a semidirect product, $\chi_{T}$ extends to $S N_{k+1}$ via the trivial representation of $S$. Again [R] says that

$$
\rho_{V}=\sigma \otimes \chi_{T}
$$

for some $\sigma \in \operatorname{Rep} S$. Using the Mackey decomposition theorem [M2] it is easy to check that since $G_{k+1} \backslash G_{k+1} N_{k+1} / S N_{k+1}$ has a single element,

$$
\left.\left(\operatorname{Ind}_{S N_{k+1}}^{G_{k+1} N_{k+1}} \sigma \otimes \chi_{T}\right)\right|_{G_{k+1}}=\operatorname{Ind}_{S}^{G_{k+1}} \sigma .
$$

In conclusion, we must show that $\sigma \in \operatorname{Rep} S, \operatorname{Ind}_{S}^{G_{k+1}} \sigma$ is $\left(\Xi_{k+1}, \operatorname{dim} \mu\right)$ bounded. This can be shown directly. However, it is easier to check this is true by using a few general facts.

Since $S \simeq F^{\times} \times F^{k}$ is solvable, $\sigma$ is weakly contained in the regular representation of $S[\mathrm{Wg}]$. Since induction is a continuous functor [F2], $\rho_{V}=\operatorname{Ind}_{S}^{G_{k+1}} \sigma$ is weakly contained in the regular representation of $G_{k+1}$. Theorem 1.9 now implies the required estimate.

The case where $k=n / 2$ is considerably easier. The group $G_{k}$ now acts simply transitively on the orbit $\mathscr{O}_{k} \subseteq \widehat{N}_{k}$. Again [R] shows that

$$
\left.\rho\right|_{G_{k} N_{k}}=\operatorname{Ind}_{N_{k}}^{G_{n} N_{k}} n \chi_{v}
$$

for any $\chi_{v} \in \mathscr{O}_{k}$ and some $n \in \mathbf{N} \cup\{\infty\}$. Again, since $G_{k} \backslash G_{k} N_{k} / N_{k}$ has a single element,

$$
\left.\rho\right|_{G_{k}}=\operatorname{Ind}_{\{1\}}^{G_{k}} n \cdot(\text { trivial })
$$

which is a multiple of the regular representation of $G_{k}$. Again Theorem 1.9 implies the desired estimate.

\section{Estimates}

We wish to use Theorem 2.2 to prove estimates on the matrix coefficients of representations of a fixed rank. Our main result will be: 
Theorem 3.1. Suppose $\rho \in \widehat{G}_{n}$ satisfies: $\left.\rho\right|_{G_{k}}$ is $\left(\Xi_{k}, \Psi\right)$-bounded for some function $\Psi$ on $\widehat{K}_{k}$. Write $n=q k+r$ with $q \in N$ and $0 \leq r<k$. If $x$ is an integer define

$$
\phi(x)= \begin{cases}x^{2} & \text { if } x \text { is even } \\ x^{2}-1 & \text { if } x \text { is odd }\end{cases}
$$

Let

$$
e(n, k)=\frac{k-1}{q k-1} \frac{\phi(q k)+\phi(r)}{\phi(n)} .
$$

Then for all $\varepsilon>0, \rho$ is $\left(\Xi_{n}^{e(n, k)-\varepsilon}, \Psi_{\varepsilon}^{\prime}\right)$-bounded for some function $\Psi_{\varepsilon}^{\prime}$ on $\widehat{K}_{n}$.

Corollary 3.2. Under the hypothesis and notation for Theorem 3.1, $\rho$ is an $L^{2 /(e(n, k)+\varepsilon}\left(G_{n} / Z\right)$-representation.

Proof. Combine Theorem 3.1 and Corollary 1.8 .

When we combine Theorem 3.1, Corollary 3.2 and Theorem 2.2 we obtain:

Theorem 3.3. Suppose $\rho \in \operatorname{Rep} G_{n}$ has pure $N$-rank $k$. If $k \neq n / 2$, then $\rho$ is $\left(\psi_{n}^{e(n, k+1)-\varepsilon}, \Psi_{\varepsilon}^{\prime}\right)$-bounded and is an $L^{2 / e(n, k+1)+\varepsilon}\left(G_{n} / Z\right)$-representation.

If $k=n / 2$, then $\rho$ is $\left(\Xi_{n}^{e(n, k)-\varepsilon}, \Psi_{\varepsilon}^{\prime}\right)$-bounded and is an $L^{2 / e(n, k)+\varepsilon}\left(G_{n} / Z\right)$ representation.

When $r=0$ (or $n=2 k-1$ ), Theorem 3.1 gives a much cleaner form for $e(n, k)$, namely $e(n, k)=(k-1) /(n-1)$. There are strong reasons to believe this estimate to be valid for all $n, k$ and not just when $k$ divides $n$. For one, the full power of the hypothesis is not quite used in the proof of Theorem 3.1 when $r \neq 0$. Furthermore, explicit calculations (e.g. for $\operatorname{GL}_{5}(\mathbf{R})$ ) show that the sharper estimate should be valid. Our theorem is not too far off, though. It is easy to see that in the worst case $(r=k-1), e(n, k)$ is about $20 \%$ smaller than $(k-1) /(n-1)$.

We formally state

Conjecture 3.4. Theorem 3.1, hence Corollary 3.2 and Theorem 3.3, are valid with $e(n, k)$ replaced by $(k-1) /(n-1)$.

We now prove Theorem 3.1. Proposition 1.6 related the asymptotics of $\Xi_{n}$ and the modular function $\delta_{B_{n}}$. During the proof we shall actually compare the various $\delta_{B}$ 's instead of the $\Xi^{\Xi}$ 's. Let $\alpha_{j}$ be the $j$ th simple root:

$$
\alpha_{j}\left(\operatorname{diag}\left(a_{1}, \ldots, a_{n}\right)\right)=a_{j+1} a_{j}^{-1}, \quad j=1, \ldots, n-1 .
$$

Then it is easy to compute

$$
\delta_{B_{n}}=\prod_{l=1}^{n-1}\left|\alpha_{l}\right|^{l(n-l)} .
$$

The proof shall proceed in steps. 
(a) First suppose $n=2 k$. If $j=0$ or 1 , let

$$
I_{j}=\{i, 0<i \leq n, i \equiv j \quad(\bmod 2)\}
$$

and $G_{k}^{(j)}=\Phi_{I_{j}}\left(G_{k}\right)$ (Definition 2.1).

We shall use superscripts ${ }^{(j)}$ for the images under $\Phi_{I_{j}}$ of all the objects defined in relations to $G_{k}$ (e.g. $\Xi_{k}^{(j)}, K_{k}^{(j)}$, etc.).

By hypothesis, $\left.\rho\right|_{G_{k}^{(j)}}$ is $\left(\Xi_{k}^{(j)}, \Psi\right)$-bounded for $j=0,1$ and some $\Psi$. Proposition 6.3 of [H1] now implies that $\left.\rho\right|_{G_{k}^{(0)} \times G_{k}^{(1)}}$ is $\left(\Xi_{k}^{(0)} \Xi_{k}^{(1)}, \tilde{\psi}\right)$-bounded for some function $\tilde{\psi}$ on $\left(K_{k}^{(0)} \times K_{k}^{(1)}\right)^{\wedge}$. (It is well known, see e.g. [H4], that $G_{n}$ and $K_{n}$ satisfy the hypotheses of this proposition.) $A_{k}^{+(0)} \times A_{k}^{+(1)}$ contains $A_{n}^{+}$. We define a function $\Phi$ on $G_{n}$ by letting it coincide with $\Xi_{k}^{(0)} \Xi_{k}^{(1)}$ on $A_{n}^{+}$and making it invariant by right and left translations by $K_{n}$.

I claim $\rho$ is $\left(\Phi, \Psi^{\prime}\right)$-bounded for some function $\Psi^{\prime}$ on $\widehat{K}_{n}$. Let $\mu$ be a $K_{n}$-type. As a representation of $K_{k}^{(0)} \times K_{k}^{(1)}, \mu=\bigoplus m_{i} \mu_{i}$, a finite direct sum of irreducible representations of $K_{k}^{(0)} \times K_{k}^{(1)}$. Define

$$
\Psi^{\prime}(\mu)=\max \tilde{\Psi}\left(\mu_{i}\right) \text {. }
$$

If $a \in A_{n}^{+}, u$ is of type $\mu$, and $v$ is of type $\nu$, then write $u=\sum u_{i}$, $v=\sum v_{j}$ corresponding to the decompositions $\mu=\bigoplus m_{i} \mu_{i}, \nu=\bigoplus n_{j} \nu_{j}$ of $\mu, \nu$ into $K_{k}^{(0)} \times K_{k}^{(1)}$-types. Then

$$
\begin{aligned}
|(\rho(a) u, v)| & \leq \sum_{i, j}\left|\left(\rho(a) u_{i}, v_{j}\right)\right| \leq \Phi(a) \sum_{i, j}\left\|u_{i}\right\|\left\|v_{i}\right\| \widetilde{\Psi}\left(\mu_{i}\right) \widetilde{\Psi}\left(\nu_{j}\right) \\
& \leq \Phi(a) \Psi^{\prime}(\mu) \Psi^{\prime}(\nu)\|u\|\|v\| .
\end{aligned}
$$

It remains to compare $\Phi$ and $\Xi_{n}$. Proposition 1.6 states that on $A_{n}^{+}$,

$$
c_{1} \delta_{B_{n}}^{-1 / 2} \leq \Xi_{n} \leq c_{2}(\varepsilon) \delta_{B_{n}}^{-1 / 2+\varepsilon}
$$

and

$$
c_{3} \delta_{B_{k}^{(0)}}^{-1 / 2} \delta_{B_{k}^{(1)}}^{-1 / 2} \leq \Phi \leq c_{4}(\varepsilon) \delta_{B_{k}^{(0)}}^{-1 / 2+\varepsilon} \delta_{B_{k}^{(1)}}^{-1 / 2+\varepsilon}
$$

for appropriate constants $c_{1}, c_{2}(\varepsilon), c_{3}, c_{4}(\varepsilon)$. Hence to finish the proof of step (a) it suffices to show

$$
\delta_{s}=\delta_{B_{k}^{(0)}} \delta_{B_{k}^{(1)}} \geq \delta_{B_{n}}^{(k-1) /(2 k-1)} .
$$

Write $\delta_{s}$ as a product of simple roots:

$$
\delta_{s}=\prod_{j=1}^{n-1}\left|\delta_{j}\right|^{s_{j}} .
$$


It is easy to compute

$$
s_{j}= \begin{cases}2 p(k-p) & \text { if } j=2 p, \\ p(k-p)+(p+1)(k-p-1) & \text { if } j=2 p+1 .\end{cases}
$$

The maximum of $(n-j) j / s_{j}$ for $j=1, \ldots, n-1$ occurs for $j=0$, $2 k-2$ and is $(2 k-1) /(k-1)$. We are comparing $\delta_{s}$ and $\delta_{B_{n}}$ in the region $\left\{a,\left|\alpha_{j}(a)\right| \geq 1\right\}$, so this is enough to prove (37). This concludes the proof of step (a).

(b) We now apply the same method to the case $n=q k, q$ arbitrary. If $j=0, \ldots, q-1$, set

$$
I_{j}=\{i, 0<i \leq n, i \equiv j(\bmod q)\} .
$$

We use superscripts ${ }^{(j)}$ in the same way as in step (a). The same argument as in that step shows that we have to compare

$$
\delta_{s}=\prod_{j=0}^{q-1} \delta_{B_{k}^{(j)}}
$$

with $\delta_{B_{n}}$. We must show

$$
\delta_{s} \geq \delta_{B_{n}}^{(k-1) /(q k-1)}
$$

If $0 \leq i<j \leq q-1$, let

$$
\delta_{i j}=\delta_{B_{k}^{(j)}} \delta_{B_{k}^{(j)}}
$$

and

$$
\delta_{i \cup j}=\delta_{B_{2 k}^{(i \cup j)}}
$$

where

$$
G_{2 k}^{(i \cup j)}=\phi_{I_{i} \cup I_{j}}\left(G_{2 k}\right)
$$

By step (a)

$$
\delta_{i j} \leq \delta_{i \cup j}^{(k-1) /(2 k-1)} .
$$

As we take the product of (46) over al pairs $i, j$ the left-hand side yields $\delta_{s}^{q-1}$. As for the right-hand side, it is easy to see that as we take the product each positive root appears only once, except for the roots in $G_{k}^{(i)}$ for some $i$. These appear $q-1$ times. Hence

$$
\delta_{s}^{q-1} \geq\left(\delta_{B_{n}} \cdot \delta_{s}^{q-2}\right)^{(k-1) /(2 k-1)},
$$

which is equivalent to (42).

(c) Now suppose $n=k+r$ and $\left.\rho\right|_{G_{k}}$ is $\left(\Xi_{k}, \Psi\right)$-bounded. If $G_{r}$ is embedded in $G_{k}$ in any standard way, it is clear from 1.6 and (31) that

$$
\left.\Xi_{k}\right|_{A_{r}^{+}}<\delta_{B_{r}}^{-\frac{1}{2}(k-1) /(r-1)+\varepsilon} .
$$


Hence for $u, v$ in the dense subspace of $K_{k}$-finite vectors, $\phi_{u, v} \in L^{2}\left(G_{r} / Z\right)$.

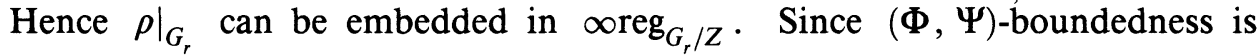
clearly preserved under taking direct sums and subrepresentations, Theorem 1.9 shows that $\left.\rho\right|_{G_{r}}$ is $\left(\Xi_{r}, \operatorname{dim} \mu\right)$-bounded.

Let

$$
I=\{[(k-r) / 2]+2 u, u=1, \ldots, r\}
$$

and let $J$ be the complement of $I$ in $\{1, \ldots, k+r\}$. Define

$$
\widetilde{G}_{r}=\phi_{I}\left(G_{r}\right)
$$

and

$$
\widetilde{G}_{k}=\phi_{J}\left(G_{k}\right) \text {. }
$$

The same argument as in step (a) shows that we have to prove

$$
\delta_{s}=\delta_{\widetilde{B}_{k}} \delta_{\widetilde{B}_{r}} \geq \delta_{B_{n}}^{(\phi(k)+\phi(r)) / \phi(n)},
$$

and again the estimate will be proved if we can show that if

$$
\delta_{s}=\prod_{i=1}^{n-1}\left|\alpha_{i}\right|^{s_{i}}
$$

then

$$
\frac{(n-i) i}{s_{i}} \leq \frac{\phi(n)}{\phi(k)+\phi(r)} .
$$

One can compute that if $n$ is even, then

$$
s_{i}=i(k-i) \text { if } i \leq(k-r) / 2,
$$

$$
s_{i}=\left\{\begin{array}{l}
\frac{1}{4}\left(k^{2}-(r-2 u)^{2}\right)+(r-u) u \text { if } i=(k-r) / 2+2 u, \\
\frac{1}{4}\left(k^{2}-(r-2 u)^{2}\right)+(r-u-1)(u+1) \text { if } i=(k-r) / 2+2 u+1
\end{array}\right.
$$

when $(k-r) / 2 \leq i \leq(k+3 r) / 2$ and

$$
s_{i}=(k+r-i)(i-r) \quad \text { if } i \geq(k+3 r) / 2 .
$$

On the other hand, if $n$ is odd,

$$
s_{i}=i(k-i) \quad \text { if } i \leq(k-r+1) / 2
$$

$$
s_{i}=\left\{\begin{aligned}
\frac{1}{4}\left(k^{2}-(r-2 u-1)^{2}\right)+(r-u) u & \text { if } i=(k-r+1) / 2+2 u, \\
\frac{1}{4}\left(k^{2}-(r-2 u-1)^{2}\right)+(r-u-1)(u+1) & \text { if } i=(k-r+1) / 2+2 u+1
\end{aligned}\right.
$$

when $(k-r+1) / 2 \leq i \leq(k+3 r+1) / 2$ and

$$
s_{i}=(k+r-i)(i-r) \quad \text { if } i \geq(k+3 r-1) / 2 .
$$


Elementary techniques now show that the maximum of $(n-i) i / s_{i}$ occurs at $i=[n / 2]$ and is $\phi(n) /(\phi(k)+\phi(r))$. This proves step (c).

(d) Finally let us prove the general case. For any standard embedding of $G_{q k}$ in $G_{n},\left.\rho\right|_{G_{q k}}$ will be $\left(\Xi^{(q k-1) /(k-1)-\varepsilon}, \Psi\right)$-bounded for some $\Psi$ by step (b). The same argument as in step (c) shows that for any standard embedding of $G_{r}$ into $G_{n},\left.\rho\right|_{G_{r}}$ is $\left(\Xi_{r}, \operatorname{dim} \mu\right)$-bounded, hence certainly $\left(\Xi_{r}^{(q k-1) /(k-1)-\varepsilon}, \operatorname{dim} \mu\right)$ bounded. Now exactly the same computations as in step (c) give the desired result, since in the proof of step (c) we just compared $\Xi$-functions. This concludes the proof of Theorem 3.1.

Our last result shows how rank may be used to derive information about the topology of $\widehat{G}_{n}$.

Theorem 3.5. Let $k \leq(n-2) / 3$ and $n>2$. Then the set $\left(\widehat{G}_{n}\right)_{k}$ of representations of $N$-rank $k$ is both open and closed in $\widehat{G}_{n}$. In particular, for $n>2$, $G_{n}$ has (a property closely related to) Kazhdan's property (T). Namely, the connected component of the trivial representation in $\widehat{G}_{n}$ consists of characters.

Proof. Since restriction defines a continuous map from $\widehat{G}_{n}$ to $\operatorname{Rep} N_{[n / 2]}$, it is obvious that $\bigcup_{l \leq k}\left(\widehat{G}_{n}\right) l$ is closed, so that it is enough to prove that under our assumptions $\left(\widehat{G}_{n}\right)_{k}$ is open. This is equivalent to showing that if $\rho \in\left(\widehat{G}_{n}\right)_{k}$ and $\sigma$ is a direct integral of representations in $\left(\widehat{G}_{n}\right)_{l}, l>k$, then $\rho$ is not weakly contained in $\sigma$.

Define a representation $\omega_{m}$ of $G_{m}$ as follows: the representation space of $\omega_{m}$ is $L^{2}\left(F^{m}\right)$. If $g \in G_{m}$ and $f \in L^{2}\left(F^{m}\right)$, we define

$$
\left(\omega_{m}(g) f\right)(x)=|\operatorname{det} g|^{1 / 2} f(x g) \text {. }
$$

Then it is obvious that under any standard embedding of $G_{l}$ in $G_{m}$,

$$
\left.\omega_{m}\right|_{G_{l}}=\infty \omega_{l} \text {. }
$$

The proof of Theorem I.3.6 shows that if $\tau \in \widehat{G}_{m}$, rk $\tau<[m / 2]$, then $\left.\tau\right|_{G_{m-2}}$ is a direct integral of representations of the form $\nu \otimes \omega_{m-2}$, with $\mathrm{rk} \nu \stackrel{m-2}{=}$ rk $\tau-1$.

An inductive argument that uses this fact and (62) shows that under our assumptions on $\rho$,

$$
\left.\rho\right|_{G_{n-2 k}}=\infty \omega_{n-2 k}^{\otimes k} .
$$

Since $n-2 k \geq k+2$, again by (62),

$$
\left.\rho\right|_{G_{k+2}}=\infty \omega_{k+2}^{\otimes k} \text {. }
$$

A straightforward calculation shows that $\omega_{m}^{\otimes l}$ is $\left(\Xi_{m}, \Phi\right)$-bounded exactly if $l \geq m-1$. Hence $\left.\rho\right|_{G_{k+2}}$ is not $\left(\Xi_{k+2}, \Phi\right)$-bounded. 
On the other hand, Theorem 2.2 and Lemma 1.4 show that $\left.\sigma\right|_{G_{k+2}}$ is $\left(\Xi_{k+2}\right.$, $\operatorname{dim} \mu$ )-bounded.

The result now follows easily from the fact that restriction to $G_{k+2}$ defines a continuous map $\widehat{G}_{n} \rightarrow \operatorname{Rep} G_{k+2}$ and from Lemma 1.5.

\section{REFERENCES}

[B] J. Bernstein, P-invariant distributions of $\mathrm{GL}(n)$ and the classification of unitary representations of $\mathrm{GL}(n)$ (non-Archimedean case), Lie Group Representations. II, Proceedings, University of Maryland, Springer, 1983, pp. 50-102.

[BZ] I. N. Bernstein and A. V. Zelevinsky, Induced representations of reductive p-adic groups. I, Ann. Sci. École Norm. Sup. (4) 10 (1977), 441-472.

[F1] J. Fell, The dual spaces of $C^{*}$-algebras, Trans. Amer. Math. Soc. 94 (1960), 364-403.

[F2] - Weak containment and induced representations of groups, Canad. J. Math. 14 (1962), 237-268.

[HC] Harish-Chandra, Discrete series for semisimple Lie groups. II, Acta Math. 116 (1966), 1-111.

[H1] R. Howe, On a notion of rank for unitary representations of the classical groups, Harmonic Analysis and Group Representations (Proc. C.I.M.E. II circlo 1980) (A. Figa-Talamanca, ed.), Liguori, 1980, pp. 223-332.

[H2] _,$\theta$-series and invariant theory, Proc. Sympos. Pure Math., vol. 33, part I, Amer. Math. Soc., Providence, R.I., 1979, pp. 275-286.

[H3] - Small unitary representations of classical groups, preprint.

[H4] - The Fourier transform and germs of characters (case of $\mathrm{GL}_{n}$ over a p-adic field), Math. Ann. 208 (1974), 305-322.

[HM] R. Howe and C. Moore, Asymptotic properties of unitary representations, J. Funct. Anal. 32 (1979), 72-96.

[K] D. Kazhdan, Connection of dual space of a group with the structure of its closed subgroups, Functional Anal. Appl. 1 (1967), 63-65.

[M1] G. Mackey, Unitary representations of group extensions. I, Acta Math. 99 (1958), 265-301.

[M2] _ Induced representations of locally compact groups. I, Ann. of Math. (2) 55 (1952), 101-139.

[R] M. Rieffel, Unitary representations of group extensions; an algebraic approach to the theory of Mackey and Blattner, Studies in Analysis (G. C. Rota, ed.), Adv. in Math. Suppl. Stud., vol. 4, Academic Press, New York, pp. 43-82.

[Sa1] S. Sahi, Spherical unitary representations of general linear groups over local fields, doctoral dissertation, Yale University, 1985.

[Sa2] _ On Kirillov's conjecture for Archimedean fields, preprint.

[S] R. Scaramuzzi, Unitary representations of small rank of general linear groups, doctoral dissertation, Yale University, 1985.

[Si] A. Silberger, Introduction to harmonic analysis on reductive p-adic groups, Princeton Univ. Press, Princeton, N.J., 1979.

[St] E. Stein, Analysis in matrix space and some new representations of $\operatorname{SL}(n, \mathbf{C})$, Ann. of Math. (2) 86 (1967), 461-490.

[T] M. Tadić, Solution of the uniterizability problem for general linear group (non-Archimedean case), preprint.

[V] D. Vogan, The unitary dual of $\mathrm{GL}(n)$ over an Archimedean field, preprint.

[VN] J. von Neumann, Die Eindeutgkeit der Schröderschen Operatores, Math. Ann. 104 (1931), 570-578.

[Wg] S. P. Wang, The dual space of semi-simple Lie groups, Amer. J. Math. 91 (1969), 921-937. 
[W] G. Warner, Harmonic analysis on semi-simple Lie groups, Springer, 1972.

[Z] G. J. Zuckerman, Continuous cohomology and unitary representations of real reductive groups, Ann. of Math. (2) 107 (1978), 495-516.

Department of Mathematics, Louisiana State University, Baton Rouge, Louisiana 70803 